\section{Zona incerta subpopulations differentially encode and modulate anxiety}

\author{
Zhuoliang Li, Giorgio Rizzi ${ }^{\dagger}$, Kelly R. Tan*
}

Despite recent clinical observations linking the zona incerta ( $\mathrm{ZI}$ ) to anxiety, little is known about whether and how the ZI processes anxiety. Here, we subject mice to anxious experiences and observe an increase in ZI c-fos-labeled neurons and single-cell calcium activity as well as an efficient effect of Zl infusion of diazepam, a classical anxiolytic drug. We further identify that somatostatin (SOM)-, calretinin (CR)-, and vesicular glutamate transporter-2 (Vglut2)expressing cells display unique electrophysiological profiles; however, they similarly respond to anxiety-provoking stimuli and to diazepam. Optogenetic manipulations reveal that each of these $\mathrm{ZI}$ neuronal populations triggers specific anxiety-related behavioral phenotypes. Activation of SOM-expressing neurons induced anxiety, while photoactivation of CR-positive cells and photoinhibition of Vglut2-expressing neurons produce anxiolysis. Furthermore, activation of CR- and Vglut2-positive cells provokes rearing and jumps, respectively. Our findings provide the first experimental evidence that $\mathrm{ZI}$ subpopulations encode and modulate different components of anxiety.

\section{INTRODUCTION}

Anxiety is a negative emotional state triggered by potential threats and generates autonomic responses, avoidance, and stress-like behaviors $(1,2)$. Several brain regions-including the amygdala (3-5), the bed nucleus of stria terminalis $(5,6)$, the medial prefrontal cortex $(7,8)$, the hippocampus $(4,8,9)$, and the hypothalamus $(9,10)$ have been reported to play an important role in anxiety. Very recently, the understudied zona incerta (ZI) has also been associated with it. Specifically, patients with Parkinson's disease (PD) treated with deep brain stimulation (DBS) in the ZI self-reported lower levels of anxiety $(11,12)$. However, there is little to no experimental evidence supporting these interesting clinical observations.

Located between the thalamus and the hypothalamus, the $\mathrm{ZI}$ is an elongated string-like structure that has been implicated in a wide range of functions, varying from neuronal development (13), hormonal regulation $(14,15)$, ingestion $(16-18)$, sleep $(19,20)$, sensory processing $(21,22)$, and pain (23). More recently, a stream of research showcased the implication of the $\mathrm{ZI}$ in emotionally related experiences and adaptive defensive behaviors. Exposure to stressful events such as sleep deprivation was shown to potentiate the expression of the immediate early gene cfos in the ZI (20). Furthermore, electrical stimulation in the ZI induced pupil dilation (24) and increased the blood flow to the extremities (24) and the heart rate (25); all of these are physiological reactions similar to that of the acute stress response (2). In addition, ZI loss of function impaired mice in their ability to respond to cues predictive of incoming threat. Specifically, mice with ZI electrolytic lesions displayed reduced avoidance in a conditioned avoidance response test (26). Similarly, ZI tetanus toxin infusion to block synaptic transmission decreased the ability to associate a predictive cue with the negative outcome in a fear conditioning task for mice (27). In contrast, optogenetic inactivation of ZI vesicular $\gamma$-aminobutyric acid (GABA) transporter (Vgat)-expressing cells during the presentations of loud tones exacerbated the triggered flight response (28). These previous studies

Biozentrum, University of Basel, Klingelbergstrasse 50/70, 4056 Basel, Switzerland. *Corresponding author. Email: kelly.tan@unibas.ch

tPresent address: Inscopix Inc., 2642 Embarcadero Way, Palo Alto, CA 94303, USA. imply that the ZI could be influenced by negative cues and modulate adaptative responses. However, how the ZI senses stressful stimuli when mice experience anxiety-triggering states is not known.

Most of the current knowledge on the ZI was gained from nonspecific manipulations, yet the ZI is a highly heterogenous structure. Histological investigations revealed diverse cell types having the capacity to release chemical signaling molecules such as glutamate, GABA, neuropeptides, and, potentially, dopamine (tyrosine hydroxylase-expressing cells) $(29,30)$. While the GABAergic neurons are abundant in number and thoroughly expressed across the ZI, the existence of somatostatin (SOM), calretinin (CR), calbindin, parvalbumin $(\mathrm{PV})$, and vasopressin indicate diversity within the GABAergic cells (29-31). Such biochemical heterogeneity may explain why the ZI has been implicated in quite a wide range of functions (30).

Here, we aimed at investigating how the ZI neuronal population responds to stressful and anxious events. On the basis of a recent report that the rostral ZI modulates cue-triggered defense behaviors (28), we speculated that the rostral ZI could also sense stressful anxiety-provoking cues. Thus, in our study, we targeted the rostral ZI (referred throughout as ZI). We first show that the ZI indeed encodes anxiety. We applied a three-step strategy with histological labeling against cfos and in vivo calcium recordings as mice experience anxious conditions, as well as the assessment of the efficacy of a pharmacological anxiety-relieving drug directly infused in the ZI. We then focused on two GABAergic subpopulations, the SOM- and CR-expressing neurons, along with the glutamatergic ones. They exhibit distinct electrophysiological properties. Although they are all activated by exposure to anxious conditions and are similarly modulated by diazepam, their bidirectional optogenetic modulation revealed distinct behavioral phenotypes. Specifically, activation of SOM-expressing cells induced anxiogenesis. Conversely, activation of CR-expressing neurons triggered anxiolysis and exploratory rearing. Last, the activation of vesicular glutamate transporter-2 (Vglut2)expressing cells induced flight-like jumps. Our data provide unprecedented information about how the ZI and some of its neuronal subpopulations encode anxiety-related behaviors. Our study hence represents an important first step into understanding the neuronal 
and circuit basis of the clinical observation for ZI DBS to relieve anxiety in patients with $\mathrm{PD}(11,12)$.

\section{RESULTS}

\section{ZI neurons encode anxiety}

On the basis of the recently reported clinical observations $(11,12)$, we first verified and assessed that the ZI encodes anxiety. We performed three experiments among different levels of investigation. First, if indeed the ZI encodes anxiety, then an anxious stimulus is expected to increase the expression of the immediate early gene cfos in the ZI. To test this hypothesis, we forced mice to experience a stay on an elevated rod, which is also exposed, meeting the two common criterions to trigger anxiety in rodents $(1,32)$. Mice were hence placed on an elevated rod $(100 \mathrm{~cm}$ above the ground and $3 \mathrm{~cm}$ in diameter; Fig. 1A) for 30 min and brought back to their home cage for an additional $30 \mathrm{~min}$ before intracardiac perfusion. Control mice stayed in their home cage for 60 min (Fig. 1B). Post hoc immunostaining against cfos (Fig. 1, C and D) followed by quantification revealed a significantly higher number of cfos-labeled neurons in the ZI of mice that experienced the elevated rod as compared to control mice (elevated rod, $3.38 \pm 0.20$ cells versus home cage, $1.00 \pm$ 0.14 cells; 951 and 243 cells, respectively, in 12 slices from three mice; Fig. 1E). These data confirm that an anxiety- or stress-triggering event increased the activity of ZI cells.

We next further investigated this with a higher temporal precision through in vivo calcium imaging. An adeno-associated virus (AAV) construct carrying the calcium indicator GCaMP6m gene was expressed in ZI neurons and a gradient refractive index (GRIN) lens implanted slightly above (Fig. 2, A and B). To investigate changes in ZI populational activity due to shifts in anxiety levels, we needed an apparatus where mice can freely explore between areas with relatively high and low anxiety-triggering values. While the elevated rod is a strong and unambiguous anxiogenic stimulus, it does not provide such a contrast. We therefore recorded the calcium dynamics of ZI neurons while mice explored an elevated platform
A

B

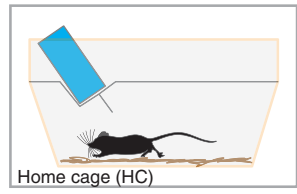

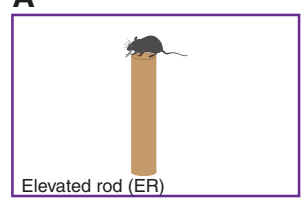

C

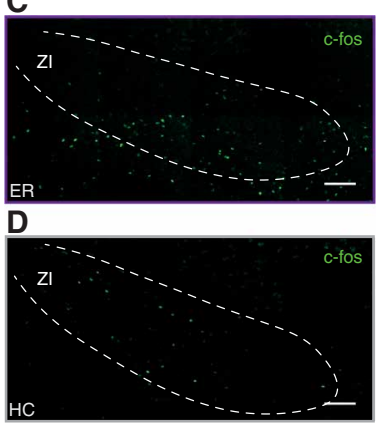

$E$

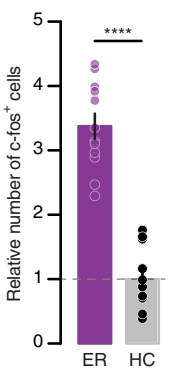

Fig. 1. Anxious experience enhances $\mathrm{ZI}$ neuronal activation. (A) Experimental setup where mice were placed on a narrow elevated rod. (B) Schematic of the control home cage condition. (C) Example confocal image of the immunohistochemical (IHC) staining against c-fos (green) in the Zl-containing coronal brain slices from mice that were exposed to the anxious elevated rod experience. Scale bar, $100 \mu \mathrm{m}$. (D) Example confocal image of the IHC labeling of c-fos in the Zl-containing coronal brain slices of mice that were left in the control home cage condition. Scale bar, $100 \mu \mathrm{m}$. (E) Bar graphs reporting the relative number of c-fos-labeled cells (normalized to the control mice) in the ZI for both experimental groups. Data are presented with means \pm SEM. ${ }^{* * * *} P<0.0001$.
(Fig. 2C), adapted from the elevated plus maze (EPM) by blocking off the closed arms. This configuration allows mice to freely explore between the relatively safer (center) and anxiety-triggering (open arms, especially the edges) areas and, at the same time, improves maneuverability of the miniscope mounted mice and maximizes the amount of time mice explored the anxiety-evoking edges. First, we observed that the mice spent more time in the center as compared to the edges (center, $48.0 \pm 3.3 \%$ versus edges, $14.3 \pm 3.3 \%$ time; Fig. 2D). This is in line with the reported values of the standard EPM (33) and hence validates that our elevated platform task evokes anxiety-like phenotypes. We observed that a substantial proportion of cells exhibited higher levels of activity when mice explored the safe center while other cells showed elevated calcium activity in the open edges (Fig. 2, E and F, and movie S1). To confirm and strengthen this observation, we further analyzed and evaluated the correlation between neuronal activity and the absolute distance of the recorded animal away from the safe center. Twenty-three percent of the imaged ZI cells displayed a positive correlation and were more active when mice explored the anxious edges as compared to the safer center (+ve Corr, $0.96 \pm 0.07$ versus not Corr, $0.06 \pm 0.04 z$ scores; 128 cells from five mice; Fig. 2, G to I). Conversely, $9 \%$ of the imaged cells exhibited a negative correlation and were less active when the animals were in the edges compared to the center (-ve Corr, $-0.75 \pm$ 0.06 versus not Corr, $0.06 \pm 0.04 z$ scores; Fig. 2 , G to I). We also analyzed the calcium dynamics as mice transitioned into a safer or more anxious location (Fig. 2, J to M). To do so, we extracted from the cells exhibiting a positive, negative, and no correlation [from Fig. 2 (G to I)]; the calcium activity immediately before and after mice transitioned into and out of the edges (edge entry and exit, respectively; Fig. 2, J and $\mathrm{K}$ ); and the center (center exit and entry; Fig. 2, L and M). On average, the positively correlated neurons increased their activity when entering the edge or leaving the center [Fig. 2], edge entry (center/transition, $0.00 \pm 0.07$ versus edge, $0.32 \pm 0.06 z$ scores); Fig. $2 \mathrm{~L}$, center exit (center, $0.00 \pm 0.03$ versus edge/transition, $0.15 \pm 0.04 z$ scores) $]$ and significantly decreased their activity when leaving the edge or entering the center [Fig. $2 \mathrm{~K}$, edge exit (edge, $0.00 \pm 0.10$ versus center/transition, $-0.36 \pm 0.03$ $z$ scores); Fig. $2 \mathrm{M}$, center entry (edge/transition, $0.00 \pm 0.03$ versus center, $-0.20 \pm 0.05 z$ scores)]. Furthermore, the magnitude of these changes was significantly larger than the not correlated cells during edge entry (+ve Corr, $0.32 \pm 0.11$ versus not Corr, $-0.07 \pm 0.05$ $z$ scores; Fig. 2J) and edge exit (+ve Corr, $-0.36 \pm 0.13$ versus not Corr, $-0.08 \pm 0.05 z$ scores; Fig. $2 \mathrm{~K}$ ) but not during center entry (+ve Corr, $-0.20 \pm 0.04$ versus not Corr, $-0.10 \pm 0.04 z$ scores; Fig. $2 \mathrm{M}$ ) or center exit (+ve Corr, $0.15 \pm 0.03$ versus not Corr, $0.10 \pm 0.04 z$ scores; Fig. $2 \mathrm{~L}$ ). On the other hand, the negatively correlated cells showed the opposite trend on average; reaching significant decreases in activity when the animals exited the edges [Fig. 2J, edge entry, (center/transition, $0.00 \pm 0.06$ versus edge, $-0.259 \pm 0.03$ $z$ scores); Fig. $2 \mathrm{~K}$, edge exit (edge, $0.00 \pm 0.03$ versus center/transition, $0.18 \pm 0.07 z$ scores); Fig. $2 \mathrm{~L}$, center exit (center, $0.00 \pm 0.03$ versus edge/transition, $-0.07 \pm 0.04 z$ scores); Fig. $2 \mathrm{M}$, center entry (edge/ transition, $0.00 \pm 0.02$ versus center, $-0.06 \pm 0.02 z$ scores)]. However, the magnitude of their responses did not different significantly from the not correlated cells [Fig. 2J, edge entry (-ve Corr, $-0.26 \pm$ 0.10 versus not Corr, $-0.07 \pm 0.05 z$ scores); Fig. $2 \mathrm{~K}$, edge exit (-ve Corr, $0.18 \pm 0.11$ versus not Corr, $-0.08 \pm 0.05 z$ scores); Fig. $2 \mathrm{~L}$, center exit ( - ve Corr, $-0.07 \pm 0.08$ versus not Corr, $0.10 \pm 0.04$ $z$ scores); Fig. $2 \mathrm{M}$, center entry (-ve Corr $-0.06 \pm 0.11$ versus not 


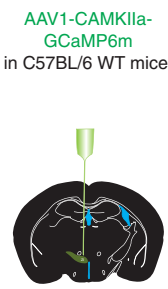

B

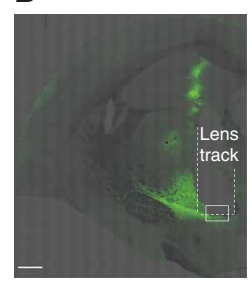
GRIN lens implant Mini-endoscope
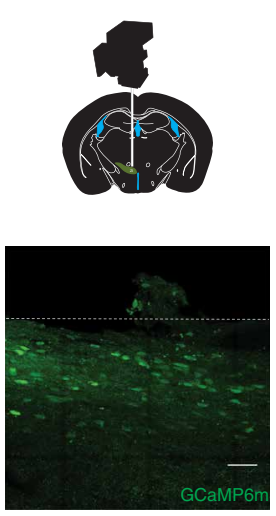

C

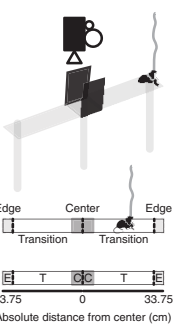

E

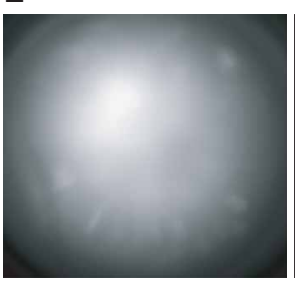

D
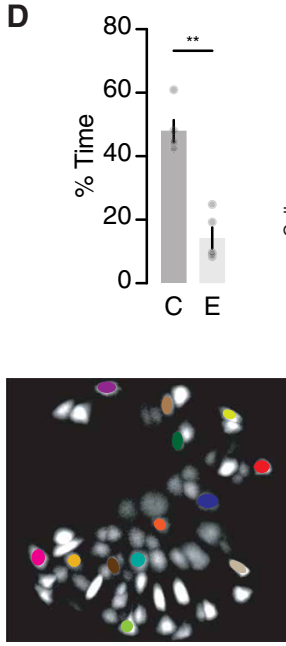
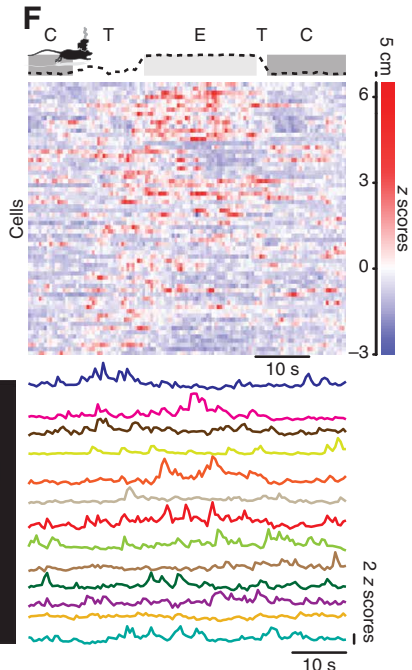

H

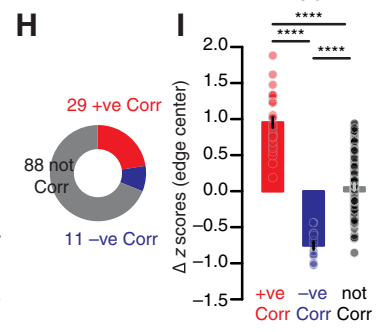

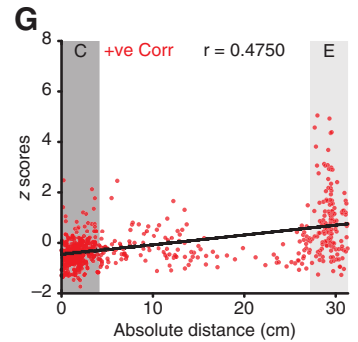
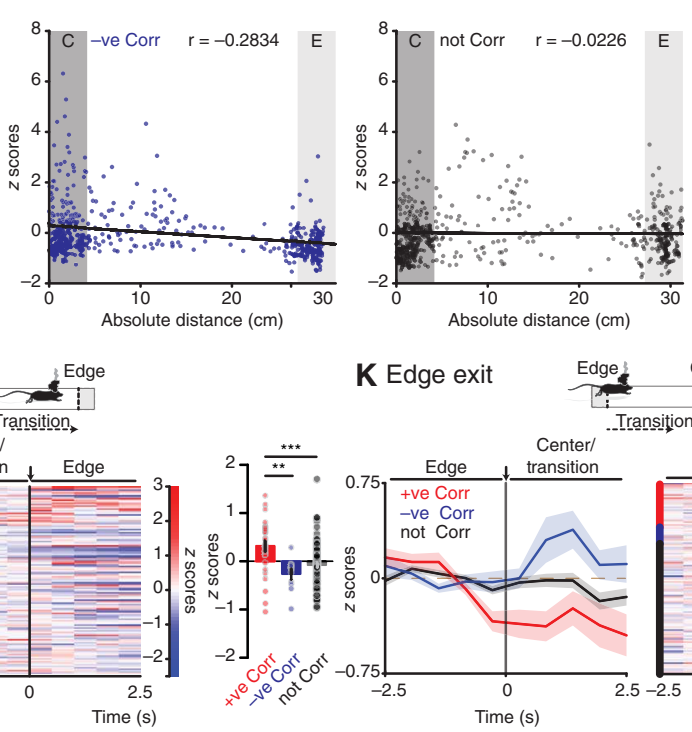

K Edge exit Edge Center
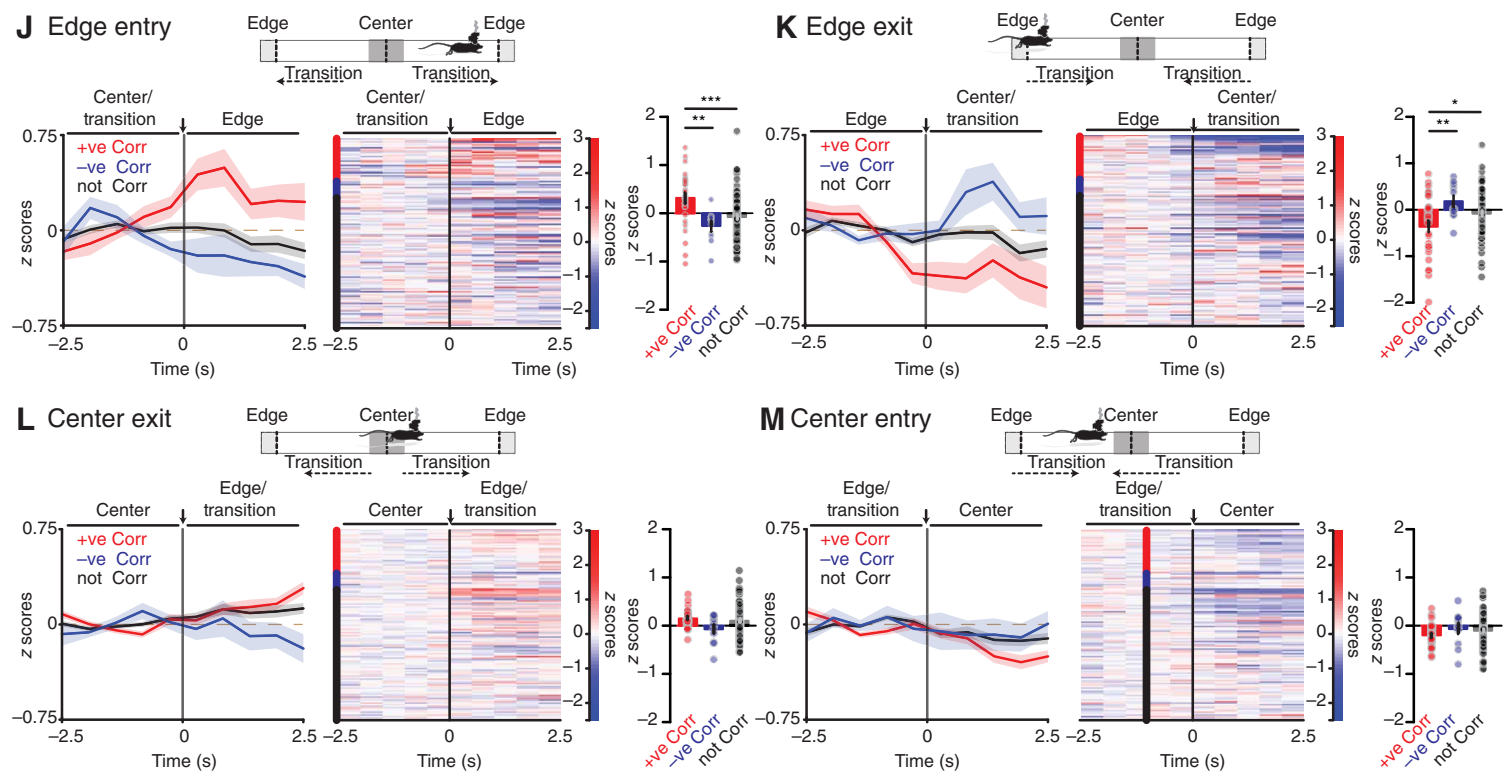

Fig. 2. ZI neurons are sensitive to anxiety-related cues. (A) Surgical setup to express and visualize GCaMP6m in the ZI of wild-type (WT) C57BL/6 mice. (B) Example confocal image of a partial coronal section containing the GRIN lens tract and the GCaMP6m-expressing (green) cells in the ZI. Scale bars, 500 and $50 \mu \mathrm{m}$. (C) Schematics of the elevated-platform design with the compartments (edge, transition, and center) annotated. (D) Bar graph reporting the percentage of time mice explored the center (C) and edges (E). (E) Raw frame extracted from a ZI calcium recording (left) and maximum intensity projection visualization of identified cells (right) with example cells color coded. (F) Track plot of a single mouse (top) along the elevated platform (different compartments highlighted). Time-locked heatmap of calcium activity (represented in $z$ scores) of all recorded cells (middle) and traces from example cells [(bottom; corresponding to (E)]. (G) Scatterplots of example recorded cells with calcium activity positively (red), negatively (blue), and not (black) correlated to the distance from the center in the recorded mouse. (H) Pie chart depicting the proportion of cells whose calcium activity correlate to the center distance. (I) Bar graphs reporting the differences in calcium activity while mice were in the edges versus the center. (J) Transient changes in activity as mice entered the edge. Schematic of the positional change (top). Line plot (left) depicting the average activity $2.5 \mathrm{~s}$ before and after the positional change. Heatmap (right) of the activity of all cells with a vertical bar depicting the type of correlation. Bar graphs (right) comparing the change in calcium activity between following the positional change. (K) Transient changes in activity as mice exited the edge. (L) Transient changes in activity as mice exited the center. (M) Transient changes in activity as mice entered the center. Data are presented with means \pm SEM. ${ }^{*} P<0.05,{ }^{* *} P<0.01,{ }^{* *} P<0.001,{ }^{* * * *} P<0.0001$. 
Corr, $-0.10 \pm 0.04 z$ scores)]. Corresponding increases and decreases in activity when mice entered more anxiety-provoking and safer areas, respectively, provide further support that this subset of ZI neurons encode anxiety-related information. To exclude a possible motor component, we correlated the calcium activity of individual ZI cells with the movement speed of the recorded mice. Of the cells that displayed positive and negative correlation with the mouse position on the platform, a majority of 69 and $91 \%$ were not correlated with the movement speed, respectively (20 of 29 and 10 of 11 cells; fig. $\mathrm{S} 1, \mathrm{~A}$ and B). On average, the speed mice that traveled in the center and the edges were similar (center, $0.038 \pm 0.003 \mathrm{~m} / \mathrm{s}$ versus edges, $0.024 \pm 0.007 \mathrm{~m} / \mathrm{s}$; fig. S1C) and thus further suggest that movement speed does not explain the differences in neuronal activity while mice were in these two areas. Together, these data reveal that a notable subset of ZI neurons is sensitive to anxiety-related cues irrespective of locomotion.

So far, we have shown that the ZI can detect changes in environments provoking relatively lower and higher levels of anxiety. We then wanted to assess the ability of the ZI to influence anxiety. We took a pharmacological approach where the anxiolytic properties of diazepam applied directly into the ZI were tested. The much smaller cannula implants posed little physical constraint on maneuverability, and thus, mice were tested in a standard paradigm for studying anxiety-like behaviors in mice (34). Of the two popular elevated mazes [the EPM and the elevated O-maze (EOM)], the EOM was selected because it allows straightforward interpretations given the simple design with equally sized safe closed and anxiogenic open arms (35). Furthermore, to ensure that a potential anxiolysis effect is not overlooked, we used BALB/c mice for their relatively heightened baseline levels of anxiety $(36,37)$. Infusion cannulas were bilaterally implanted above the ZI (Fig. 3, A and B). Diazepam or vehicle infusions were made 30 min before placing mice in the EOM (Fig. 3C). It was previously reported that diazepam ( $10 \mathrm{mg} / \mathrm{kg}$, provided intraperitoneally) was anxiolytic without affecting general locomotion (38), and thus, we provided a dose 10 times smaller for infusion. The order of the treatments was counterbalanced and reversed 2 weeks later. When mice received diazepam, they spent significantly more time in the open arms as compared to when they were treated with the vehicle (vehicle, $130.8 \pm 27.85 \mathrm{~s}$ versus diazepam, $219.9 \pm 30.03 \mathrm{~s} ; 13$ mice; Fig. 3, D and E, and movie S2). They also made more entries into
A

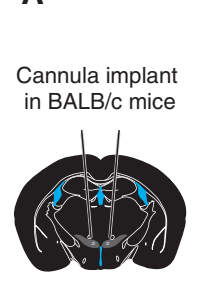

D

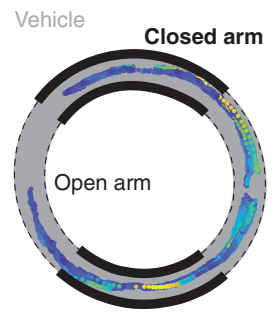

G

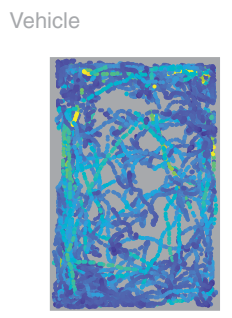

B
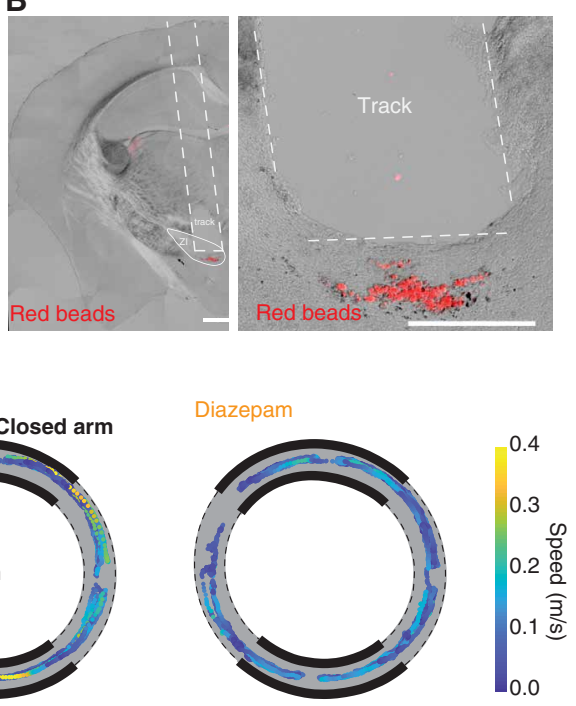

0.0
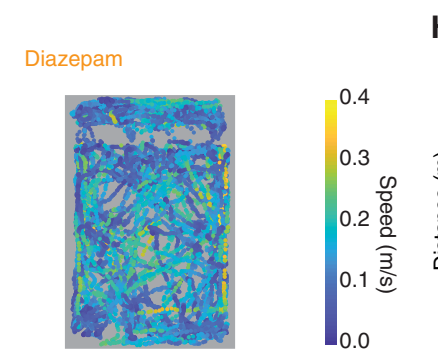

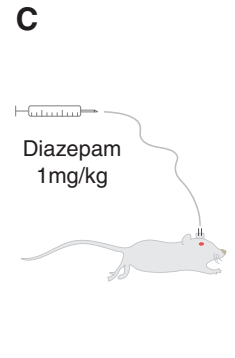

E

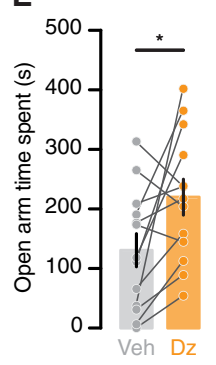

H

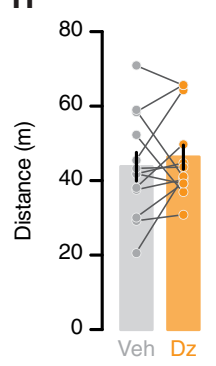

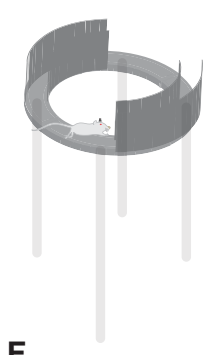

$\mathbf{F}$

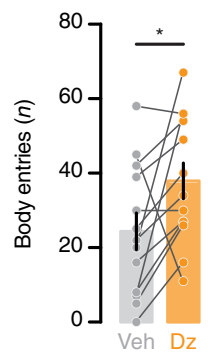

I

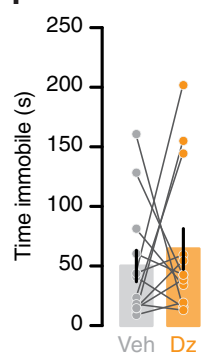

Fig. 3. Local ZI diazepam infusion reduces anxiety without affecting movement. (A) Surgical setup to place cannulas above the Zl. (B) Example confocal image of a partial coronal section containing the cannula tract with the red RetroBeads in the Zl to show the extent of spread. Scale bars, 500 and $250 \mu \mathrm{m}$. (C) Schematic of the setup to directly infuse diazepam (1 mg/kg) into the Zl or vehicle $30 \mathrm{~min}$ before testing the mouse on the EOM. (D) Track plot of a single example mouse on the EOM (dashed lines denote the open arms, while the filled lines indicate the closed arms) after vehicle (left) and diazepam (right) infusion with the speed color coded. (E) Bar graph of time the mice spent in the open arms after vehicle and diazepam infusions. (F) Bar graph depicting the number of times mice entered the open arm after vehicle and diazepam infusions. (G) Track plot of an example mouse in the OFT after vehicle (left) and diazepam (right) infusion with the speed color coded. (H) Bar graph depicting the distance mice traveled after vehicle or diazepam infusions. (I) Bar graph showing the time mice spent immobile after vehicle or diazepam infusions. Data are presented with means \pm SEM. ${ }^{*} P<0.05$. 
the open arms (Fig. 3F, vehicle $24.38 \pm 4.90$ entries versus diazepam $37.92 \pm 4.77$ entries). In addition, we observed no significant differences across distance and immobility measures between diazepamand vehicle-treated mice in the EOM (fig. S2). To make sure that the diazepam-mediated anxiolytic effects in the EOM were not cofounded by changes in locomotion, we investigated general locomotion with the open field test (OFT). The distance traveled and the time mice spent immobile in the OFT were similar with both treatment conditions (distance traveled: vehicle, $43.76 \pm 3.82$ versus diazepam, $46.33 \pm 3.22$; time immobile: vehicle, $50.05 \pm 13.17$ s versus diazepam, $64.39 \pm 17.09$ s; Fig. 3, G to I). These findings suggest that local diazepam treatment in the ZI reduces anxiety without affecting locomotion, further consolidating our biochemical and in vivo calcium imaging data. To briefly summarize here, our multilevel experimental observations converge onto the ZI as a brain structure processing anxiety-related stimulus and modulating exploration into anxiety-evoking environments.

\section{ZI SOM-, CR-, and Vglut2-expressing cells exhibit unique electrophysiological profiles and are all sensitive to anxious stimuli and diazepam.}

So far, we have shown with complementary approaches that the ZI encodes anxiety. However, note that we observed subsets of ZI neurons displaying anxiety-induced cfos expression and anxietyinduced changes in calcium transients. Previous literature reported the cellular diversity within the ZI (29-31). We therefore wondered about the specific role that an individual ZI subpopulation plays in the encoding of anxiety. To address this question, we first examined the ZI biochemical diversity with fluorescent in situ hybridization (FISH), focusing on the GABAergic and glutamatergic markers with Vgat and Vglut2, respectively. We report that Vgat-expressing cells are more abundant than Vglut2-expressing ones (Vgat-only, 78.8\% and Vglut2-only, 19.0\%; 3390 cells from three mice; Fig. 4, A and B). Probing against specific inhibitory markers, namely, SOM, CR, and $\mathrm{PV}$, revealed that the SOM- and CR-expressing subpopulations are larger than the PV-expressing one (SOM-only, 36.6\%; CR-only, 53.2\%; and PV-only, 2.0\%; 1684 cells from three mice; Fig. 4, C and D). As expected, SOM- and CR-expressing cells coexpress Vgat (fig. S3).

We also investigated the coexpression of calcium/calmodulindependent protein kinase II $\alpha$ (CaMKII $\alpha$ ) with $\mathrm{SOM}^{-}$and $\mathrm{CR}^{-}$(fig. S4, A to F) or with Vglut2 $^{-}$(fig. S4, G to K) in the ZI in separate preparations. We observed that subsets of CaMKII $\alpha$-expressing cells also coexpress SOM- (16.3\%), CR- (26.4\%), or Vglut2- (19.7\%) (fig. S4, A to K). With these data, we confirmed that the imaged neurons (GCaMP6m under the expression of CaMKII $\alpha$ promoter) recorded in Fig. 2 indeed included SOM-, CR-, and Vglut2-expressing cell types.

Considering that SOM- and CR-expressing cells represent a sizable portion of the Vgat-positive neurons (as opposed to the PV-expressing cells; Fig. 4 and fig. S3), we sought to focus the study onto these two inhibitory subpopulations and the rare excitatory Vglut2-expressing one.

Next, we functionally characterized these ZI subpopulations performing in vitro whole-cell patch-clamp recordings (Fig. 4, E to M). To unequivocally study SOM-, CR-, and Vglut2-expressing cells, reporter lines (SOM-TOM and CR-TOM) or CRE-dependent EYFP (enhanced yellow fluorescent protein; AAV5-DIO-EYFP) that injected Vglut2-CRE-mouse line was used (Fig. 4, E and F). Despite exhibiting similar capacitance (SOM, $14.27 \pm 0.80 \mathrm{pF}$; CR, $16.94 \pm$ $2.16 \mathrm{pF}$; and Vglut2, $15.15 \pm 1.09 \mathrm{pF} ; 13$ to 16 cells; four to five mice;
Fig. 4G), resting membrane potential (SOM, $-72.20 \pm 2.12 \mathrm{mV}$; $\mathrm{CR},-74.31 \pm 3.37 \mathrm{mV}$; and Vglut $2,-68.15 \pm 2.88 \mathrm{mV}$; Fig. $4 \mathrm{H})$, and action potential width (SOM, $8.46 \pm 0.16 \mathrm{~ms}$; CR, $8.46 \pm 0.13 \mathrm{~ms}$; and Vglut2, $9.16 \pm 0.47 \mathrm{~ms}$; Fig. 4I), SOM-expressing neurons are less excitable and display a higher rheobase (SOM, $41.33 \pm 5.68 \mathrm{pA}$; $\mathrm{CR}, 23.13 \pm 2.54$ pA; and Vglut2 $29.17 \pm 3.362$ pA; Fig. 4J) while Vglut2-expressing cells sustain higher maximum firing frequencies $(\mathrm{SOM}, 45.43 \pm 5.34 \mathrm{~Hz}$; CR, $42.00 \pm 6.38 \mathrm{~Hz}$; and Vglut2, $105.20 \pm$ $11.42 \mathrm{~Hz}$; Fig. 4, K to M).

To characterize further these three neuronal populations in a circuit mapping context, we first assessed their local connectivity probability and, in a second step, their long-range anatomical mapping. We injected an AAV vector containing CRE-dependent ChR2 (Channelrhodopsin-2) (AAV5-DIO-ChR2-EYFP) in the ZI of CR-, SOM-, and Vglut2-CRE mice (fig. S5A). We performed in vitro electrophysiology experiments in brain slices containing the ZI and recorded from ChR2-negative cells identified by the lack of a 400-ms blue $(473 \mathrm{~nm})$ light-induced desensitizing current. Local postsynaptically connected cells exhibited a brief 5-ms light-induced current response. We observed no ( 0 of 20 patched cells) locally connected cells to the SOM-expressing subpopulation (fig. S5B). In contrast, we observed $21.5 \%$ connected cells to the CR-expressing subpopulation (fig. S5D) and $59 \%$ connected cells to the Vglut $2^{-}$subpopulation (fig. S5F). These connections are all monosynaptic, as challenged by the successive bath application of tetrodotoxin (TTX) and 4-aminopyridine (4-AP), and are inhibitory and excitatory, as abolished by subsequent bath application of picrotoxin (PTX) and kynurenic acid (KA), respectively (fig. S5, D to G).

We next identified SOM-, CR-, and Vglut2-expressing cells' long-range projections to other brain structures. To do so, we injected an AAV construct containing CRE-dependent tdTOM and synaptophysin-tagged enhanced green fluorescent protein (SypEGFP) into the ZI of SOM-, CR-, and Vglut2-CRE mice and comprehensively explored the diversity of the efferent projections across the brain (fig. S6). Across all three ZI subpopulations, the most noticeable tdTOM and SypEGFP signals were observed in the lateral habenula (LHb) and the periaqueductal gray (PAG) (fig. S6). While CRexpressing ZI cells sparsely project to the dorsal medial PAG (dmPAG), the Vglut2-expressing cells densely project to the lateral and ventrolateral PAG (1/vlPAG), and the SOM-expressing cells project to all three (fig. S6).

We next assessed whether SOM-, CR-, and Vglut2-expressing ZI neurons are activated by anxious conditions. The elevated rod experiment followed by cfos labeling was performed (fig. S7). Our quantification reports that coexpression of cfos with either $\mathrm{SOM}^{-}$, $\mathrm{CR}^{-}$, or Vglut $2^{-}$is significantly increased in the experimental mice as compared to the control ones (SOM: elevated rod, $3.50 \pm 0.35$ cells versus home cage, $1.00 \pm 0.18$ cells; 3262 and 2458 cells in total, respectively, in 12 slices from three mice; CR: elevated rod, $7.40 \pm 1.39$ cells versus home cage, $1.00 \pm 0.25$ cells; 2074 and 1624 cells in total, respectively; Vglut2: elevated rod, $3.28 \pm 0.30$ versus home cage, $1.00 \pm 0.17$ cells; 2538 and 1551 cells in total, respectively; fig. S7, $\mathrm{B}$ to $\mathrm{P})$. These data suggest that SOM-, CR-, and Vglut2-expressing cells are all activated by anxiety.

Last, considering that the intra-ZI infusion of diazepam induced anxiolysis, we sought of verifying the sensitivity of our three neuronal cell types to diazepam. This was done through in vitro recordings of pharmacologically isolated inhibitory postsynaptic currents (IPSCs) onto SOM-, CR-, and Vglut2-expressing cells. The neurons 
A
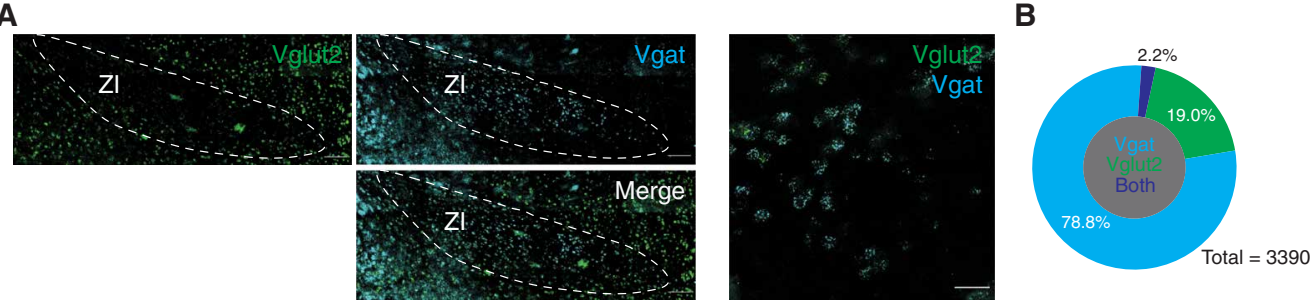

C
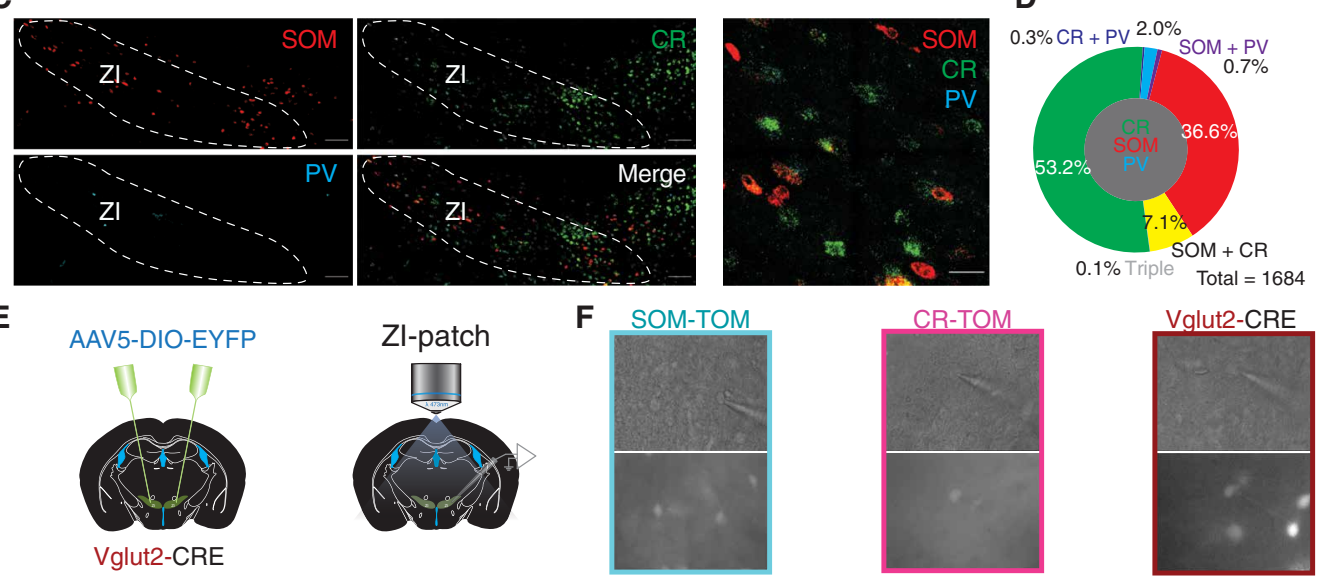

E
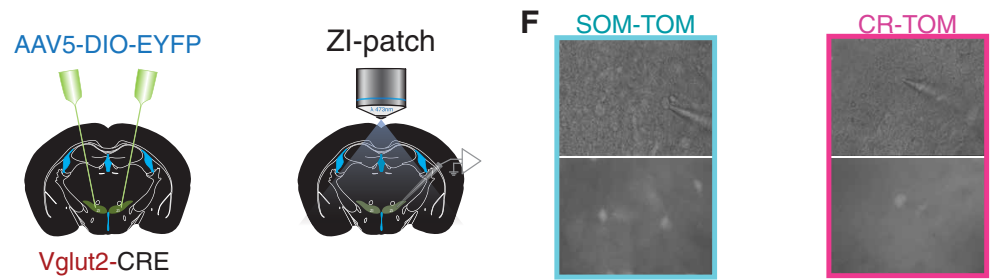

or SOM-TOM and CR-TOM
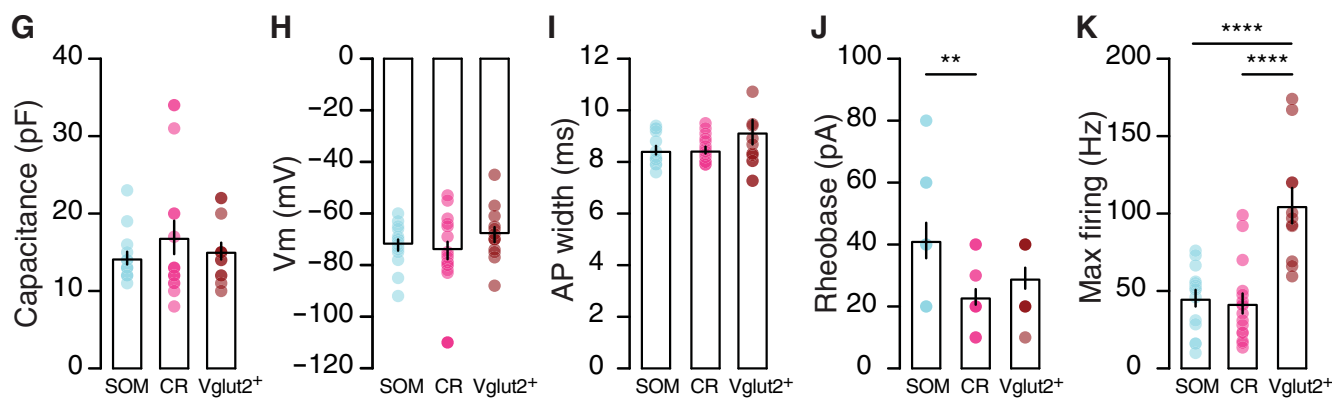
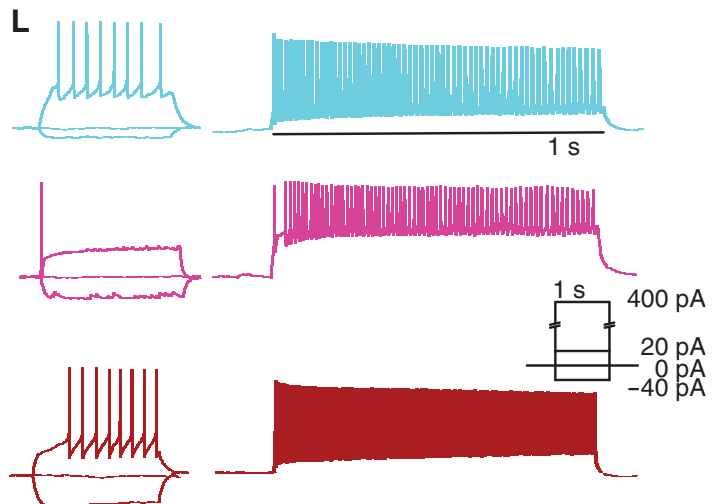

M

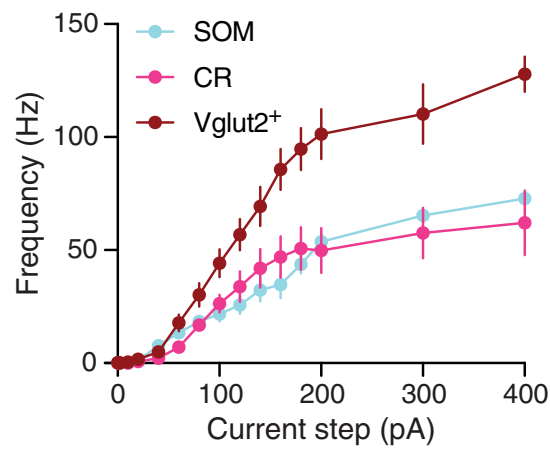

Fig. 4. SOM-, CR-, and Vglut2-expressing ZI neurons display unique electrophysiological properties. (A) Example confocal images of Zl-containing coronal brain slices with the FISH labeling of the mRNAs for Vglut2 (green) and Vgat (cyan) and the merged image. Scale bars, 100 and $25 \mu \mathrm{m}$. (B) Pie chart quantification of the proportion of cells in ZI expressing Vglut2, Vgat, or both (3390 cells total, three mice). (C) Example confocal images of the Zl-containing coronal brain sections with the FISH labeling of the mRNAs for SOM (red), CR (green), PV (cyan), and the merged. Scale bars, 100 and $25 \mu \mathrm{m}$. (D) Pie chart representation of the percentage of expressing the mRNAs for SOM, CR, PV, or any combination of the three (1684 cells total, three mice). (E) Schematic of the experimental procedure. (F) Example bright-field (top) and fluorescent (bottom) images of patched cells: SOM (cyan), CR (magenta), and Vglut2 (maroon). (G) Bar graphs reporting the capacitance from SOM-, CR-, and Vglut2-expressing ZI cells. (H) Bar graphs depicting the resting membrane potential (Vm) from SOM-, CR-, and Vglut2-expressing ZI cells. (I) Bar graphs of the action potential width from SOM-, CR-, and Vglut2-expressing ZI cells. (J) Bar graphs presenting the rheobase from SOM-, CR-, and Vglut2-expressing ZI cells. (K) Bar graphs summarizing the maximum firing frequency from SOM-, CR-, and Vglut2-expressing ZI cells. (L) Example current clamp recordings from SOM-, CR-, and Vglut2-expressing ZI cells upon various current step injections. (M) Summary input/output curve for SOM-, CR-, and Vglut2-positive cells. Data are presented with means \pm SEM. ${ }^{* *} P<0.01,{ }^{* * * *} P<0.0001$. 
were identified through the expression of TdTomato/EGFP (Fig. 5A). Diazepam $(1 \mu \mathrm{M})$ potentiated the electrically evoked IPSCs (SOM: baseline, $-523.9 \pm 96.29 \mathrm{pA}$ versus diazepam, $-907.7 \pm 175.3 \mathrm{pA}$; CR: baseline, $-232.9 \pm 63.65 \mathrm{pA}$ versus diazepam, $-500.3 \pm 85.55 \mathrm{pA}$; Vglut2: baseline, $-342.0 \pm 71.26 \mathrm{pA}$ versus diazepam, $-669.0 \pm 106.5 \mathrm{pA}$; 10 to 11 cells from four to five mice; Fig. 5B) without affecting the pair-pulse ratio in all three cell types (SOM: baseline, $1.11 \pm 0.09$ versus diazepam, $0.91 \pm 0.07$; CR: baseline, $0.95 \pm 0.11$ versus diazepam, $0.85 \pm 0.08$; Vglut2: baseline, $1.06 \pm 0.07$ versus diazepam, $0.98 \pm 0.06$; Fig. $5 B$ ). The amplitude (SOM: baseline, $-41.78 \pm 5.07 \mathrm{pA}$ versus diazepam, $-48.65 \pm 6.27 \mathrm{pA}$; CR: baseline, $-44.54 \pm 3.05 \mathrm{pA}$ versus diazepam, $-50.08 \pm 3.12 \mathrm{pA}$; Vglut2: baseline, $40.79 \pm 3.22 \mathrm{pA}$ versus diazepam, $-47.54 \pm 3.98 \mathrm{pA} ; 10$ to 12 cells from four to five mice; Fig. $5 \mathrm{C}$ ) and frequency (SOM: baseline, $0.05 \pm 0.02 \mathrm{~Hz}$ versus diazepam, $0.09 \pm 0.03 \mathrm{~Hz}$; CR: baseline, $0.04 \pm 0.01 \mathrm{~Hz}$ versus diazepam, $0.06 \pm$ $0.01 \mathrm{~Hz}$; Vglut2: baseline, $0.08 \pm 0.02 \mathrm{~Hz}$ versus diazepam $0.09 \pm 0.02 \mathrm{~Hz}$;
Fig. 5C) of the spontaneous IPSCs were both increased. The IPSCs were blocked by PTX $(100 \mu \mathrm{M})$. These in vitro electrophysiological data reveal that all three neuronal subpopulations are sensitive to diazepam.

Together, these findings suggest that SOM-, CR-, and Vglut2expressing cells of the ZI are distinct neuronal subpopulations with specific functional intrinsic properties. However, they are all similarly activated by anxious stimuli and are sensitive to diazepam.

\section{SOM-, CR-, and Vglut2-expressing ZI neurons trigger distinct anxiety-related traits}

Despite being activated by elevation and allosterically modulated by diazepam, SOM-, CR-, and Vglut2-expressing cells might still engage different components of anxiety-related behaviors. To verify this hypothesis, we bidirectionally manipulated the activity of these three subpopulations while mice performed the EOM task (Fig. 6).

A

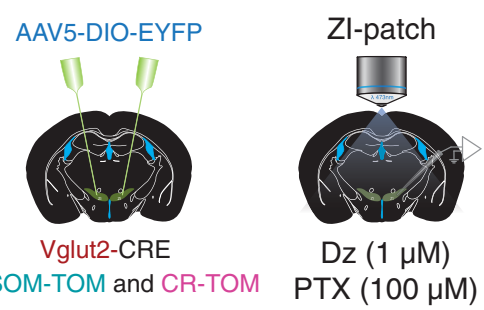

B
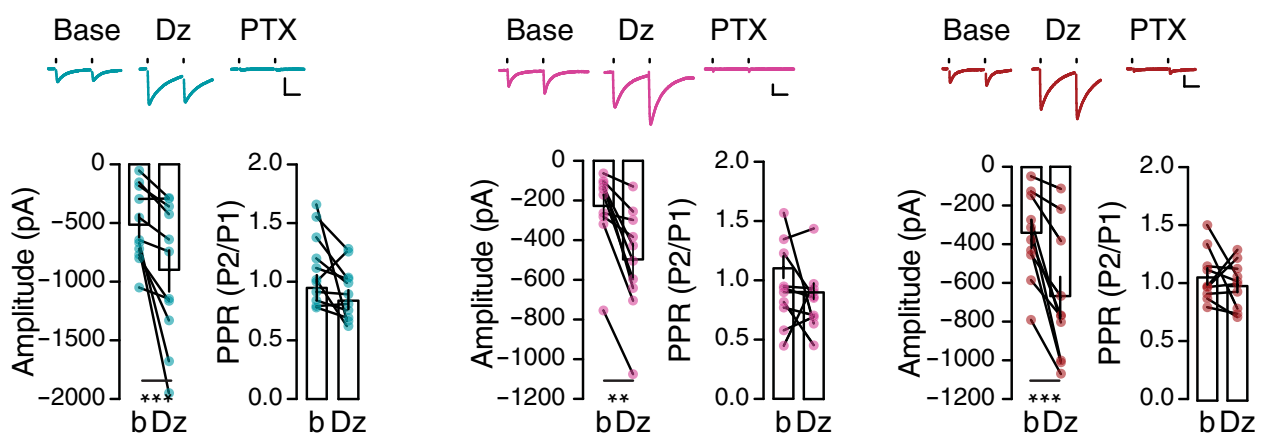

C
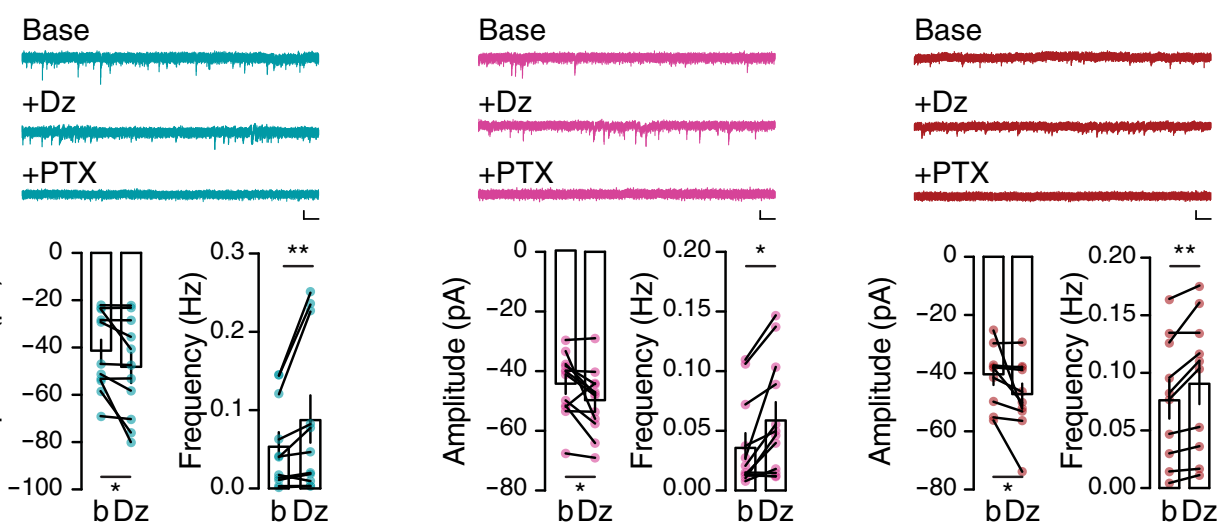

Fig. 5. Diazepam potentiates inhibitory drive onto SOM-, CR-, and Vglut2-expressing ZI neurons. (A) Schematic of the experimental setup. (B) Example IPSC from representative SOM-expressing (cyan), CR-expressing (magenta), and Vglut2-expressing (maroon) ZI cells during baseline (base) and following subsequent bath application of diazepam (Dz) and PTX. Scale bars, 100 pA (vertical) and $25 \mathrm{~ms}$ (horizontal). Bar graphs report the amplitude of the IPSCs and the paired-pulse ratio (PPR) of both conditions. (C) Example sIPSCs (spontaneous inhibitory post-synaptic currents) recorded from representative SOM-, CR-, and Vglut2-expressing Zl cells during baseline (base) and during bath application of diazepam (Dz) and PTX. Scale bars, $30 \mathrm{pA}$ (vertical) and $500 \mathrm{~ms}$ (horizontal). Bar graphs of the amplitude and the frequency of sIPSCs in the baseline and following diazepam application are shown. Data are presented with means \pm SEM. ${ }^{*} P<0.05,{ }^{* *} P<0.01,{ }^{* * *} P<0.001$. 
A

AAV5-DIO-ChR2-EYFP

or AAV5-DIO-Arch-EYFP Bilateral implants or AAV5-DIO-EYFP
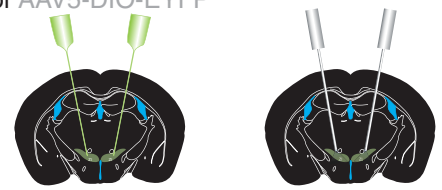

SOM- or CR- or Vglut2-CRE

C

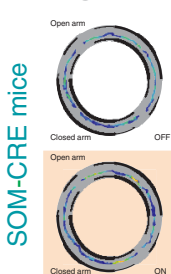

ChR2

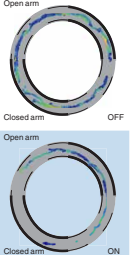

E

D

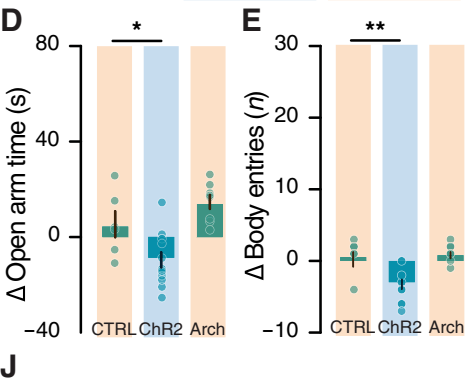

AAV5-DIO-ChR2-EYFP Bilateral implants

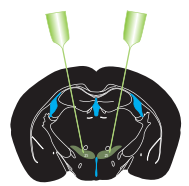

CR- Or
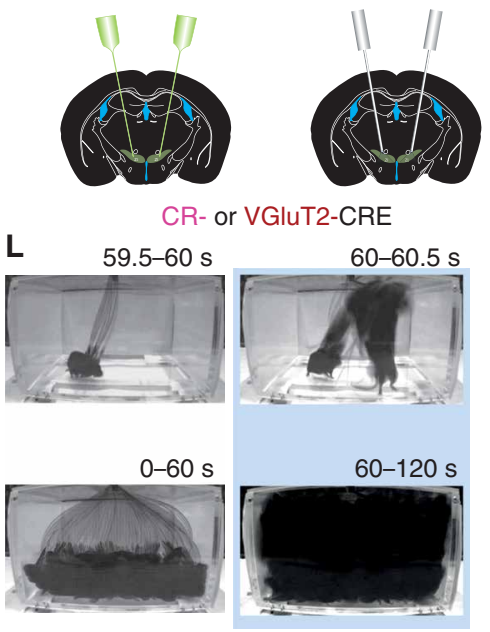
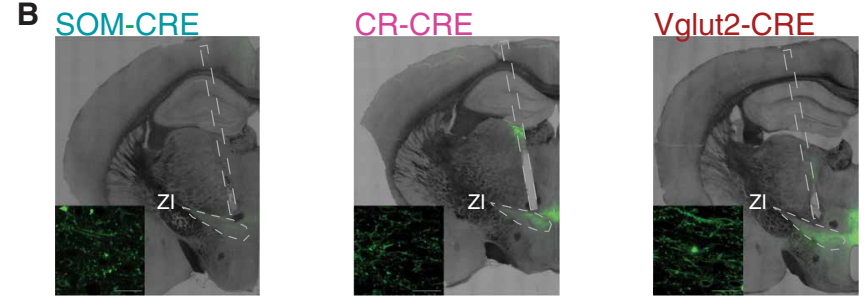

$473 \mathrm{~nm}, 10 \mathrm{~mW} @ 40 \mathrm{~Hz}, 5-\mathrm{ms}$ pulses OFF ON OFF ON OFF ON

532 nm, 10 mW @ continuous light OFF ON OFF ON OFF ON
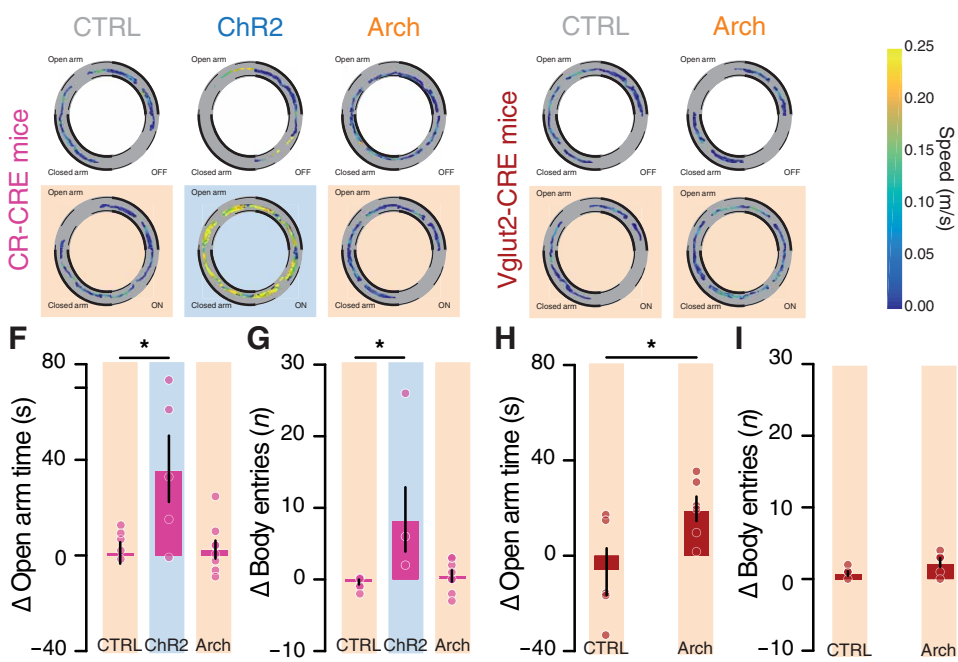

$\mathrm{K}$

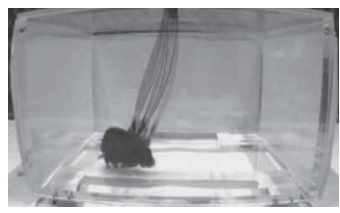

$473 \mathrm{~nm}, 10 \mathrm{~mW} @ 40 \mathrm{~Hz}, 5-\mathrm{ms}$ pulses OFF ON OFF ON OFF ON
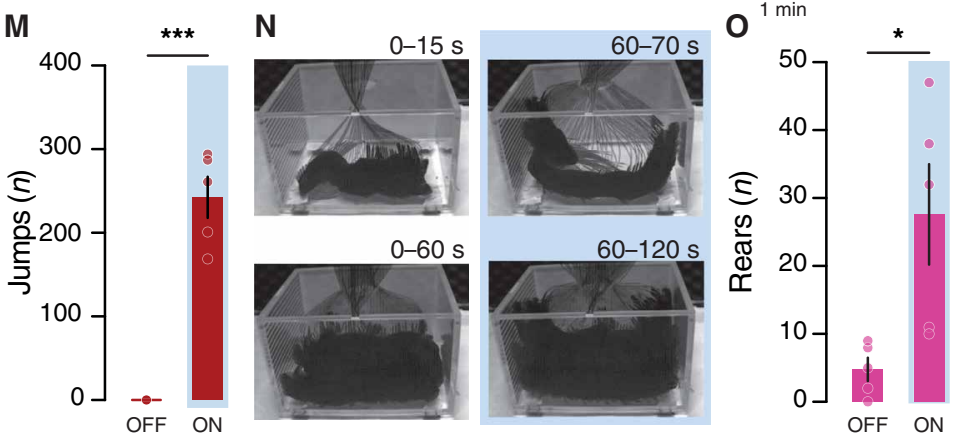

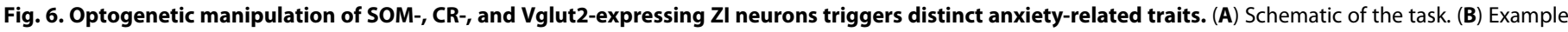

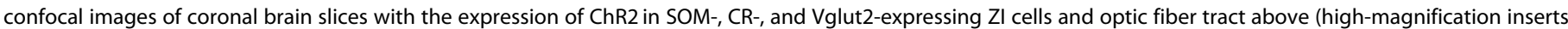

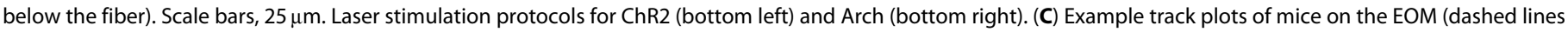

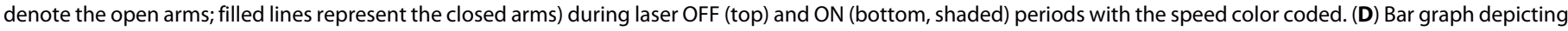

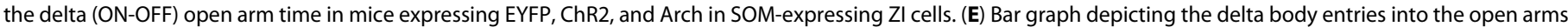

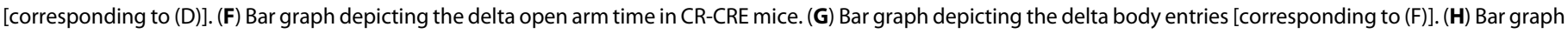

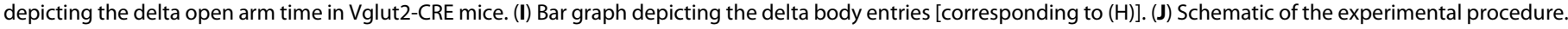

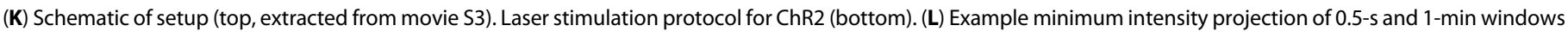

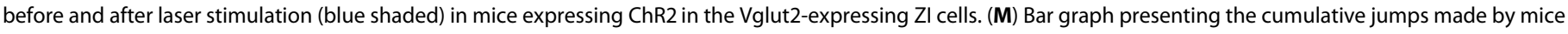

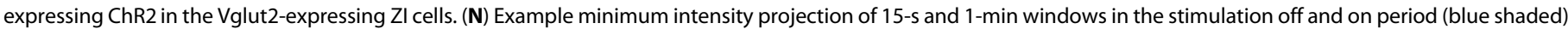

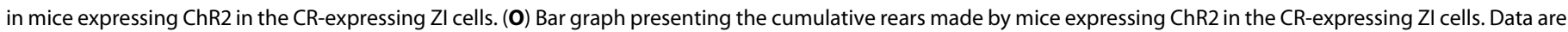
presented with means \pm SEM. ${ }^{*} P<0.05,{ }^{* *} P<0.01,{ }^{* *} P<0.001$. Photo credit: Zhuoliang Li, University of Basel. 
To do so, we injected AAV vectors containing either CRE-dependent ChR2 (AAV5-DIO-ChR2-EYFP) or Arch (AAV5-DIO-Arch-EYFP) in the ZI of SOM-, CR-, and Vglut2-CRE mice and implanted optic fibers above the injection site (Fig. 6, A and B). Our experimental preparations were validated through in vitro patch-clamp recordings (fig. S8, A and B). A short blue light illumination (400 ms, $473 \mathrm{~nm}$ ) evoked desensitizing photocurrents in ChR2-positive SOM-, CR-, and Vglut2-positive ZI neurons (fig. S8C). Furthermore, either a 1-s continuous light pulse or a $10-\mathrm{s}(40 \mathrm{~Hz})$ stimulation elicited firing activity that was reversed as soon as the stimulation was turned off (fig. S8D). Such a $40-\mathrm{Hz}$ blue light stimulation was efficient at significantly and sufficiently enhancing the firing activity of each three cell types (fig. S8D). In contrast, green light pulses (400 ms, $532 \mathrm{~nm}$ ) triggered hyperpolarizing photocurrents in Arch expressing SOM-, CR-, and Vglut2-positive cells, preventing evoked or spontaneous firing (fig. S8, E and F).

At the behavioral level, mice performed the EOM task while the activity of SOM-, CR-, and Vglut2-expressing cells was manipulated ( 1 min off and 1 min on; three times; Fig. 6C). Behavioral changes induced by light stimulation were reported as the difference in the performance during $\mathrm{ON}$ and OFF light stimulation. Activation of ChR2 in SOM-expressing ZI neurons significantly reduced both the amount of time spent in the open arm (CTRL, $5.40 \pm 5.47 \mathrm{~s}$ versus ChR2, $-9.53 \pm 3.16 \mathrm{~s} ; 6$ to 12 mice; Fig. 6D) and the number of entries made into the open arm (CTRL, $0.83 \pm$ 1.01 entries versus ChR2, $-3.26 \pm 0.65$ entries; Fig. $6 \mathrm{E})$. In stark contrast, optogenetic activation of CR-expressing neurons induced an opposite behavioral phenotype, with a significant increase of both the time spent in the open arm (CTRL, $1.98 \pm 4.47 \mathrm{~s}$ versus ChR2, $36.32 \pm 13.83 \mathrm{~s}$; five to eight mice; Fig. $6 \mathrm{~F}$ ) and the number of open arm entries $(-0.67 \pm 0.33$ entries versus $8.40 \pm 4.49$ entries; Fig. 6G). The optogenetic inhibition of either SOM- or CR-positive ZI neurons had little effect on the EOM performance [Fig. 6D, SOM open arm time (CTRL, $5.40 \pm 5.47 \mathrm{~s}$ versus Arch, $14.74 \pm$ 2.89 s); Fig. 6E, SOM open arm entry (CTRL, $0.83 \pm 1.01$ entries versus Arch, $1.13 \pm 0.44$ entries); Fig. $6 \mathrm{~F}, \mathrm{CR}$ open arm time (CTRL, $1.98 \pm 4.47$ s versus Arch, $3.38 \pm 3.74$ s); Fig. 6G, CR open arm entry $(-0.67 \pm 0.33$ entries versus $0.50 \pm 0.78$ entries $)]$. The lightinduced inactivation of Vglut2-expressing cells increased the time mice spent in the open arms (CTRL, $-6.64 \pm 9.80 \mathrm{~s}$ versus Arch, $19.75 \pm 5.14 \mathrm{~s}$; five to six mice; Fig. $6 \mathrm{H}$ ) without affecting the number of open arm entries (CTRL, $1.00 \pm 0.32$ entries versus Arch, $2.33 \pm 0.62$ entries; Fig. 6I).

When mice were tested in the open field, the same light stimulations induced no significant change in locomotion (fig. S9), as reported by the distance traveled [fig. S9D, SOM (CTRL, $-0.34 \pm 0.35 \mathrm{~m}$ versus ChR2, $-1.82 \pm 0.63 \mathrm{~m}$ versus Arch, $0.40 \pm 0.57 \mathrm{~m}$ ); fig. S9F, CR (CTRL, $-0.50 \pm 0.67 \mathrm{~m}$ versus ChR2, $-0.03 \pm 1.15 \mathrm{~m}$ versus Arch, $0.78 \pm 0.65 \mathrm{~m}$ ); fig. S9H, Vglut2 (CTRL, $-0.27 \pm 0.65 \mathrm{~m}$ versus Arch, $1.42 \pm-0.83 \mathrm{~m}$ )] and the immobility time [fig. S9E, SOM (CTRL, $-6.22 \pm$ $6.90 \mathrm{~s}$ versus $\mathrm{ChR} 2,26.2 \pm 10.29 \mathrm{~s}$ versus Arch, $-2.51 \pm 9.26 \mathrm{~s})$; fig. S9G, CR (CTRL, $-7.22 \pm 9.77$ s versus ChR2, $8.28 \pm 11.05$ s versus Arch, $-16.24 \pm 8.11 \mathrm{~s}$ ); fig. S9I, Vglut2 (CTRL, $11.18 \pm 6.94 \mathrm{~s}$ versus Arch, $-24.52 \pm 14.03 \mathrm{~s}$ )]. Photoactivation of Vglut2-expressing neurons was also tested. Because of the phototriggered impressive jumps, testing mice in the EOM and the OFT was not possible. To measure this phenotype, mice were placed inside a closed transparent chamber (Fig. 6, J and K). Blue light stimulation $(40 \mathrm{~Hz})$ triggered time locked jumps that were immediately stopped when the light stimulation was OFF (OFF, $0.00 \pm 0.00$ jumps versus $\mathrm{ON}, 242.4 \pm$ 24.60 jumps; Fig. 6, L and M, and movie S3).

To verify that our light stimulation is restricted to Vglut2expressing ZI cells, we conducted and reproduced the jumping phenotype in a separate cohort of Vglut2-CRE mice (off, $0.00 \pm 0.00$ jumps versus on, $179.7 \pm 56.81$ jumps; fig. S10, A to C) and post hoc stained slices containing the implant site against cfos. We observed that while cfos staining was sparsely observed in the ventral thalamus or the lateral hypothalamus, the majority of the cfos-positive neurons were concentrated within the ZI together with the ChR2-eYFP labeled cells (fig. S10D).

Last, considering that ZI CR-ChR2-expressing mice exhibited an increased exploration in the EOM, we also tested them in the closed transparent chamber. Light stimulation significantly increased the number of rears $(\mathrm{OFF}, 4.80 \pm 1.72$ rears versus $\mathrm{ON}, 27.60 \pm 7.38$ rears; Fig. 6, $\mathrm{N}$ and $\mathrm{O}$, and movie $\mathrm{S} 4$ ). This exploratory rearing behavior $(39,40)$ provides an additional support that the activation of CRexpressing ZI neurons reduces anxiety. Furthermore, the activation of SOM-expressing neurons induced no clear-cut observable behavioral alterations (movie S5).

Together, our findings suggest that SOM-, CR-, and Vglut2expressing ZI subpopulations differentially modulate anxiety traits and related behaviors. Specifically, activation of SOM-expressing ZI neurons induces anxiogenesis, while the activation of CR-expressing ZI neurons triggers anxiolysis and exploratory rears. Activation of Vglut2-expressing ZI neurons induces jumps and is contrasted by its inactivation, which is anxiolytic.

\section{DISCUSSION}

With this study, we aimed at providing experimental evidence that the ZI encodes anxiety, in light of the interesting recent clinical observations reporting that ZI DBS treatment in PD reduces anxiety $(11,12)$. Specifically, we showed that a powerful anxiety-provoking stimulus such as the forced exposure on a narrow elevated rod activates $\mathrm{ZI}$ neurons, as reported by their significant enhancement in cfos expression, adding to the growing literature that the ZI is sensitive to stressful experiences (20). Given the temporal limitations of such a biochemical investigation, we scrutinized the same question by examining the in vivo calcium dynamics of individual ZI neurons with a calcium indicator expressed under a CaMKII $\alpha$ promoter while mice explored an elevated platform. We revealed that a subset of ZI neurons is active in the open arm edges and become more active when mice enter a more anxiogenic area and less to a safer one. These attributes cannot be solely explained by changes in motion, as most movement speed-correlated ZI cells are not correlated with anxiety. Supplementing this, the local infusion of a classical anxiolytic drug, diazepam directly into the ZI, reduced anxiety without affecting locomotion on the EOM and OFT, respectively.

Note that not all ZI cells show potentiated cfos expression or increased calcium activity following exposure to anxiogenic cues. These observations hint toward some underlying heterogeneities. Past literature provided comprehensive insights into the diversity of biochemical cell type markers expressed in the ZI (29-31), without much further characterization into their physiological and functional properties. In line with previous studies $(29,31)$, we showed that cells expressing GABAergic markers are more prevalent over glutamatergic ones. We focused on two GABAergic subpopulations expressing Vgat with SOM or CR in addition to the rarer 
Vglut2-expressing cells and reported their unique intrinsic properties, leading the way with the electrophysiological profiling of genetically defined ZI subpopulations. Each of them coexpress CaMKII $\alpha$. SOM-expressing cells seem to not modulate local network activity, whereas CR- and Vglut2-expressing neurons do act as interneurons, providing local inhibitory and excitatory modulations onto other ZI cells, respectively. Anatomically, all three ZI subpopulations send long-range projections innervating brain structures involved in emotionally relevant behaviors $(2,41)$. Not only did we confirm the pathways ZI onto the LHb $(42)$ and the PAG $(27,28)$, but we also delineate further that while CR-expressing cells innervate the dmPAG, Vglut2-positive cells project onto the 1/vlPAG and SOMexpressing neurons project onto all three.

We also provide additional experimental evidence showing that they similarly respond to anxiety-related cues biochemically and to diazepam ex vivo; however, they drive specific and distinct actions. Optogenetic activation of SOM-expressing cells reduced exploration in the anxiogenic open arms, while the activation of CR-expressing neurons and inhibition of Vglut2-expressing ZI cells increased it. These findings differ from previous reports where manipulation of the ZI did not influence anxiety. Specifically, nonselective inhibition of ZI neuronal activity with either tetanus toxin or chemogenetic manipulations was reported to not significantly affect exploration in the EPM (27) and OFT (43). Moreover, chemogenetic inhibition of Vgat-expressing neurons did not reduce anxiogenic center exploration in the OFT (43). These differences could potentially derive from the compensatory mechanisms following permanent impairments of the ZI by the tetanus toxin in the former study and the mild nature of center in the OFT to provoke anxiety by the latter investigation or due to differences in the specificity of cells manipulated in both cases.

In addition, we observed that the photoactivation of Vglut2expressing ZI cells induced time-locked jumps that were rapid, directed toward the walls of the chamber, and continuously changing in direction. It is tempting to associate these light-induced jumps with compulsive repetitive jumping behavior observed in a genetic model of autism spectrum disorder (44) or in mice undergoing kindling seizures (45) given the multitude of jumps within a small period of time in all three cases. However, the jumps that we observed following the stimulation of Vglut2-positive ZI cells were not stationary but rather directed as an attempt to escape and continuously change its direction once they fail to do so. The lack of additional motor disturbances before jumping further argues against the notion that these light-induced jumps were consequences of a seizure. Furthermore, the directed nature of these jumps resembles the active flight behavior observed in mice presented with an auditory tone that was previously paired with a strong aversive footshock (46). A recent report showed that the excitation of vlPAG promotes flight behavior (47). We show that ZI Vglut2-expressing cells do project onto the vlPAG; hence, we speculate that the observed flight-like jumps may be driven by the Vglut2-expressing ZI cells to vlPAG subpathway. On the other hand, the activation of CRexpressing ZI cells promoted rearing, a rodent-specific exploratory behavior $(48,49)$ that is reduced in anxious animals following stressful experiences while elevated in animals with low anxiety states $(39,40)$. Together with the anxiolytic effects observed in the EOM, the activation of CR-expressing ZI cells could provide the necessary foundations and partake in foraging (18) and hunting (50) behaviors, actions that have been previously attributed to the
ZI. Last, these behavioral alterations in anxiety-related behaviors that we observed with the optogenetic manipulations of its cell types are not concomitant with any significant changes in general locomotion, as is similarly reported by others $(27,28,43)$.

While we bidirectionally manipulated the activity of SOM-, CR-, and Vglut2-expressing ZI neurons, the behavioral modifications were often unidirectional. The inability for the inhibition of these cell types to produce significant opposing modulations in anxiety may potentially be attributed to the insufficient drive by Arch to completely shut down ongoing activity for prolonged periods of time in face of strong excitatory inputs.

Photoactivation of SOM- and CR-expressing cells triggered opposite behavioral phenotype. This could potentially be due to the differences in their outgoing projections or underlying local connectivity. More specifically, it is tempting to speculate that CRexpressing cells may inhibit SOM-positive neurons, hence modulating and shifting the overall ZI dynamic to affect behavior. However, a much elaborated and systematic investigation into the ZI local connectivity including the identification of both the pre- and postsynaptic cells would be necessary to infer any circuit mechanisms.

Overall, our experimental findings support our hypothesis that the ZI plays an important role in anxiety. Through changes in the expression of activity-dependent biochemical markers and neuronal activity, subsets of ZI cells detect changes in the environment, triggering low and potentiated anxiety states. However, the ZI does not only have a sensory role but also actively influence anxietyrelated behaviors, namely, the exploration of exposed and elevated areas, when pharmacologically targeted. Through specific anatomical connections, ZI neuronal subpopulations engage in distinct actions to potentially generate a global anxiety-related behavioral response. Specifically, the activation of SOM-expressing neurons promotes avoidance of anxiogenic areas, while the Vglut2-positive cells induce flight-like jumps. In stark contrast, the activation of CR-expressing cells drives exploration into anxiety-provoking environments and also encourages rearing. Together, these findings provide converging evidence that the $\mathrm{ZI}$ and its neuronal subpopulations can sense anxiety-related information and influence anxiety-related states and behavioral outcomes.

Building on the clinical observations, our findings provide important experimental evidence to show that the ZI and its individual subpopulations encode anxiety and guide related behaviors. Our study hence provides valuable knowledge regarding the role of the ZI in anxiety and extends a positive outlook for the ZI as a therapeutic target for the treatment of anxiety.

\section{MATERIALS AND METHODS Animals}

Male and female SOM-CRE (B6-Sst<tm2.1(cre)Zjh $>/$ J) (51), CRCRE (B6(Cg)-Calb2<tm1(cre)Zjh>/J) (51), Vglut2-CRE (B6.CgSlc17a6<tm2(cre)Lowl $>$ Unc/J, UNC) (52), and C57BL/6JRj mice were bred in house. SOM-TOM and CR-TOM reporter lines were generated by crossing the respective Cre lines with the Ai9 transgenic line [B6;129S6-Gt(ROSA)26Sor $<$ tm9(CAG-tdTomato)Hze $>/ J]$ (53). All experimentation performed with transgenic mice involved mice of both sexes. No clear sex differences were observed, and the data were thus pooled (fig. S11). BALB/cByJRj mice, previously shown to have elevated levels of anxiety $(36,37)$, were purchased from Janvier Labs. All experimental procedures were approved by the 
Institutional Animal Care Office of the University of Basel and the Cantonal Veterinary Office under the license number 2742.

\section{Surgery \\ General}

Mice were anesthetized with gas isoflurane (5\% for induction and $1.5 \%$ for maintenance) in $\mathrm{O}_{2}$ (Provet/Primal Healthcare, EZ Anesthesia Systems) and placed onto the stereotaxic frame (World Precision Instruments). Lidocaine $(0.2 \mathrm{mg} / \mathrm{g})$ (Streuli Pharma) was injected subcutaneously above the skull. The skin was disinfected with 70\% ethanol and Betadine (Mundipharma). A small incision along the anterior-posterior axis was made at the midline, and then, the skull was leveled. A small hole was drilled through the skull above the ZI, which consists of the regions ZI and subincertal nucleus in the mouse atlas $(1.3 \mathrm{~mm}$ posteriorly to the bregma, $\pm 1.3 \mathrm{~mm}$ laterally to the midline, and $-4.87 \mathrm{~mm}$ deep from the surface of the skull at a $10^{\circ}$ angle away from the midline) (54). Glass micropipettes (Drummond) were pulled with a vertical puller (Narishige). Viral vectors were ejected into the ZI with a pressure injector system controlled by a pulse generator (A.M.P.I.; 10 to $100 \mathrm{~ms}, 20$ psi, at $0.333 \mathrm{~Hz}$ ). The wound was either stitched together with tissue absorptive silk sutures (SABANA) or continued with additional implantations (optic fiber, cannula, or GRIN lens). Buprenorphine (Bupaq P, Streuli Pharma AG) pain killers were administered $(0.1 \mu \mathrm{g} / \mathrm{g})$ after surgery as needed.

\section{Calcium imaging}

Viruses (AAV1.Camk2a.GCaMP6m.WPRE.SV40, Ready-to-Image virus, Inscopix) were unilaterally (150 nl) injected in the $\mathrm{ZI}$, and then, a GRIN lens (Inscopix; $600 \mu \mathrm{m}$ wide and $7.3 \mathrm{~mm}$ long) was placed at the same coordinates. The lens was fixed with an ultraviolet (UV)-light curable glue (Henkel). A custom-made head bar $(2 \mathrm{~cm}$ long, $0.4 \mathrm{~cm}$ wide, and $0.1 \mathrm{~cm}$ tall) was placed for future handling. A fixed headcap was built from layers consisting of superglue (Cyberbond), UV-light curable glue (Loctite), and dental cement (Lang). Small screws were anchored into the skull to improve adhesion between the skull and the headcap. The headcap was secured to the skin with Vetbond tissue adhesive glue (3M). The implant was covered with temporary silicone gels (KauPo Smooth-On). The expression of the GCaMP6m and the clearing of the lens were assessed regularly starting from 2 weeks after surgery. Once the conditions were visually determined to be satisfactory, the mice were placed under isoflurane anesthesia on the stereotaxic frame and the baseplate was secured to the headcap using a UV-light curable flowable composite glue (Kerr).

\section{Cannula implantation}

Custom guide cannulas ( $460 \mu \mathrm{m}$ in diameter and $5.5 \mathrm{~mm}$ in length; P1 Technologies) were bilaterally placed above the ZI. The cannulas were filled with a dummy cannula of the same length and covered by temporary silicone gel (KauPo).

\section{Diazepam infusion}

Following surgical recovery ( $>2$ weeks), internal cannulas $(100 \mu \mathrm{m}$ in inner diameter and $5.5 \mathrm{~mm}$ in length; P1 Technologies) compatible with the guide cannulas were inserted. Freshly prepared diazepam ( $1 \mathrm{mg} / \mathrm{kg}$; Fagron) in vehicle (50\% propylene glycol in sterile deionized $\mathrm{H}_{2} \mathrm{O}$; Sigma-Aldrich) or vehicle were bilaterally infused into the ZI with the syringe pump (Harvard Apparatus) at $100 \mathrm{nl} / \mathrm{min}$. The treatments were reversed after 2 weeks. The long gap is implemented to avoid potential acute reexposure effects on anxiety-related behaviors in the behavioral paradigms involving elevated mazes $(55,56)$.
Following the end of all behavioral experiments, Red RetroBeads (Lumafluor) were infused via the same injection system for anatomical validation. The mice were then sacrificed, and histological preparations (see the "Histology" section) were made.

\section{Preparation of reporter mice for cfos screening or in vitro recordings}

To identify Vglut $2^{+} \mathrm{ZI}$ neurons while a reporter line is lacking, adult male and female (8 to 10 weeks) Vglut2-CRE mice were bilaterally injected with a virus expressing a CRE-dependent fluorescent protein (AAV5-EF1 $\alpha$-DIO-EYFP, UNC Vector Core; $300 \mathrm{nl}$ per side). The virus was expressed for at least 2 weeks before histology or in vitro electrophysiology experiments.

\section{Anatomical tracing}

Mice were injected with viruses that contained a CRE-dependent tdTOM with a synaptophysin-tagged EGFP (AAV1-phSyn-FLEXtdTom-T2A-SypEGFP, UNC Vector Core; $100 \mathrm{nl}$ per side). The virus was expressed for at least 3 weeks before perfusion and histology.

\section{Optogenetics and fiber implantation}

Mice were injected with viruses that contained a CRE-dependent opsin construct (AAV5-EF1 $\alpha$-DIO-eArch3.0-EYFP and AAV5-EF1 $\alpha$ DIO-ChR2-H134R-EYFP; UNC/UZH Vector Cores) or a control fluorescent protein (AAV5-EF1 $\alpha$-DIO-EYFP; UNC Vector Core) bilaterally in the 5ZI (150 $\mathrm{nl}$ per side). Optic fibers [ $>70 \%$ light transmittance, $200 \mu \mathrm{m}$ in diameter, and 0.39 numerical aperture (NA) optic fibers fixed by epoxy to 1.25 -mm-wide 6.4 -mm-long ceramic ferrules; Thorlabs] (57) were bilaterally placed above the ZI $(250 \mu \mathrm{m})$.

\section{In vitro electrophysiology}

Coronal mouse brain slices (180 $\mu \mathrm{m}$ thick) containing the ZI were collected with a vibratome (Leica) in cooled artificial cerebrospinal fluid (ACSF) cutting solution (92 mM N-methyl-D-glucamine, $2.5 \mathrm{mM} \mathrm{KCl} 1.25 \mathrm{mM} \mathrm{NaH}_{2} \mathrm{PO}_{4}, 30 \mathrm{mM} \mathrm{NaHCO}_{3}, 20 \mathrm{mM}_{\text {Hepes, }}$ $25 \mathrm{mM}$ glucose, $2 \mathrm{mM}$ thiourea, $5 \mathrm{mM} \mathrm{Na}$-ascorbate, $3 \mathrm{mM} \mathrm{Na}$ pyruvate, $0.5 \mathrm{mM} \mathrm{CaCl} 2 \cdot 2 \mathrm{H}_{2} \mathrm{O}, 10 \mathrm{mM} \mathrm{MgSO} \cdot \cdot 7 \mathrm{H}_{2} \mathrm{O}$, and $10 \mathrm{mM}$ NAC ( $N$-Acetyl-L-cysteine); osmolarity of 290 to 300 mosM) bubbled with $95 \% \mathrm{O}_{2}$ and $5 \% \mathrm{CO}_{2}$. The slices were kept in the holding ACSF solution [ $92 \mathrm{mM} \mathrm{NaCl}, 2.5 \mathrm{mM} \mathrm{KCl}, 1.25 \mathrm{mM} \mathrm{NaH}_{2} \mathrm{PO}_{4}, 30 \mathrm{mM}$ $\mathrm{NaHCO}_{3}, 20 \mathrm{mM}$ Hepes, $25 \mathrm{mM}$ glucose, $2 \mathrm{mM}$ thiourea, $5 \mathrm{mM}$ Na-ascorbate, $3 \mathrm{mMNa}$-pyruvate, $2 \mathrm{mM} \mathrm{CaCl}_{2} \cdot 2 \mathrm{H}_{2} \mathrm{O}, 2 \mathrm{mM} \mathrm{MgSO}{ }_{4} \cdot 7 \mathrm{H}_{2} \mathrm{O}$, and $10 \mathrm{mM} \mathrm{NAC} \mathrm{(pH} \mathrm{7.3);} \mathrm{osmolarity} \mathrm{of} 290$ to $300 \mathrm{mosM}$ ] at $31^{\circ} \mathrm{C}$ before being transferred into the recording chamber containing the recording ACSF solution [119 $\mathrm{mM} \mathrm{NaCl}, 2.5 \mathrm{mM} \mathrm{KCl}, 1.25 \mathrm{mM}$ $\mathrm{NaH}_{2} \mathrm{PO}_{4}, 24 \mathrm{mM} \mathrm{NaHCO} 3,12.5 \mathrm{mM}$ glucose, $2 \mathrm{mM} \mathrm{CaCl} \cdot 2 \mathrm{H}_{2} \mathrm{O}$, and $2 \mathrm{mM} \mathrm{MgSO}_{4} \cdot 7 \mathrm{H}_{2} \mathrm{O}$ ( $\mathrm{pH} 7.3$ ); osmolarity of 290 to $300 \mathrm{mosM}$ ] bubbled with $95 \% \mathrm{O}_{2}$ and $5 \% \mathrm{CO}_{2}$. Visualized whole-cell patch recordings were performed to record cells from the ZI. We identified SOM-, CR-, and Vglut2-positive cells by the expression of TdTomato from reporter lines or virally introduced fluorescent proteins (AAV5-EF1 $\alpha$-DIO-EYFP, AAV5-EF1 $\alpha$-DIO-eArch3.0-EYFP, and AAV5-EF1 $\alpha$-DIO-ChR2-H134R-EYFP) in each of the three cell typespecific CRE lines (SOM-CRE, CR-CRE, and Vglut2-CRE) visualized under an upright microscope (Olympus) through PLANFL $\mathrm{N} \times 4$ and $\times 40$ (Olympus) objectives. The internal solution contained the following: for electrophysiological profiling and optogenetic tools validation, $130 \mathrm{mM}$ K-gluconate, $10 \mathrm{mM}$ creatine phosphate, $4 \mathrm{mM} \mathrm{MgCl}_{2}$, $3.4 \mathrm{mM} \mathrm{Na} \mathrm{A}_{2} \mathrm{ATP}, 0.1 \mathrm{mM} \mathrm{Na}{ }_{3} \mathrm{GTP}, 1.1 \mathrm{mM}$ EGTA, and $5 \mathrm{mM}$ Hepes (pH 7.3); osmolarity of 289 mosM; for IPSC recordings and recording inhibitory transmission, $30 \mathrm{mM} \mathrm{K}$-gluconate, $100 \mathrm{mM} \mathrm{KCl}$, 
$10 \mathrm{mM}$ creatine phosphate, $4 \mathrm{mM} \mathrm{MgCl}_{2}, 3.4 \mathrm{mM} \mathrm{Na}_{2} \mathrm{ATP}, 0.1 \mathrm{mM}$ $\mathrm{Na}_{3}$ GTP, $1.1 \mathrm{mM}$ EGTA, and 5 mM Hepes (pH 7.3); osmolarity of $289 \mathrm{mosM}$; and for recording excitatory transmission, $130 \mathrm{mM} \mathrm{CsCl}$, $4 \mathrm{mM} \mathrm{NaCl}, 5 \mathrm{mM}$ creatine phosphate, $2 \mathrm{mM} \mathrm{MgCl}_{2}, 2 \mathrm{mM}$ $\mathrm{Na}_{2} \mathrm{ATP}, 0.6 \mathrm{mM} \mathrm{Na}_{3} \mathrm{GTP}, 1.1 \mathrm{mM}$ EGTA, and $5 \mathrm{mM}$ Hepes ( $\mathrm{pH} 7.3)$; osmolarity of 290 mosM.

To investigate the ex vivo effect of diazepam, diazepam $(1 \mu \mathrm{M}$; Fagron) was subsequently washed in and blocked with PTX $(100 \mu \mathrm{M})$. In separate slices, spontaneous inhibitory postsynaptic potentials were recorded and also subsequently bath applied with diazepam and blocked with PTX. For the validation of optogenetic tools, light flashes ( $400 \mathrm{~ms}$ ) controlled by a pulse generator (A.M.P.I) were delivered from a microscope mounted light-emitting diode (CoolLED) to evoke synaptic currents in voltage clamp mode held at resting membrane potential. To study the local connectivity, brief ( $5 \mathrm{~ms}$ ) light pulses (50 ms apart) were delivered to evoke postsynaptic currents in non-ChR2-expressing cells. The concentrations of the pharmacological agents were applied at $500 \mathrm{nM}$ (TTX), $300 \mu \mathrm{M}$ (4-AP), $100 \mu \mathrm{M}$ (PTX), and $2 \mathrm{mM}$ (KA). In current clamp mode, blue light flashes ( $1 \mathrm{~s}$ continuous and $10 \mathrm{~s}$ at $40 \mathrm{~Hz}$ ) were used to evoke firing in ChR2-expressing cells and orange light flashes (1 s continuous) were used to reduce electrically evoked (current injected) firing.

\section{Histology \\ Immunohistochemistry}

Mice received a lethal dose of pentobarbital $(0.3 \mathrm{mg} / \mathrm{kg}$, intraperitoneally) (Streuli Pharma AG). Mice were fixed onto a Styrofoam board (with the back facing the board and the chest facing outward) with 25-gauge needles (Braun) by the skin of their paws. The fixed mice are then placed at approximately $45^{\circ}$. While clenching the chest skin with forceps, a vertical incision is made with surgical scissors (Fine Science Tools) to remove the outer skin. An incision is then made to expose, and another is made to break open the diaphragm. Fat deposits around the heart are carefully removed with forceps. A small cut is made with a finer scissor to tear open the right atrium. The animals were then transcardially perfused with $25 \mathrm{ml}$ of phosphate-buffered saline (PBS) buffer (Sigma-Aldrich) followed by the same volume of $4 \%$ paraformaldehyde (PFA; Sigma-Aldrich) in PBS through the left ventricle. Brains were extracted and postfixed in $4 \%$ PFA overnight then immersed in $30 \%$ sucrose in PBS until the brains have completely sunk to the floor of the container (complete absorption of sucrose). Sections were prepared $(50 \mu \mathrm{m})$ using a cryostat (Leica CM1950). Brain slices containing the ZI slices were first washed (three times for $3 \mathrm{~min}$ ) with $1 \times$ tris-buffered saline (TBS) (Sigma-Aldrich) with $0.1 \%$ Tween 20 (Sigma-Aldrich), permeabilized for 20 min with $1 \times$ TBS with $0.1 \%$ Tween 20 and Triton X-100 (Sigma-Aldrich), and blocked for $120 \mathrm{~min}$ with $1 \%$ bovine serum albumin (Sigma-Aldrich) or 1\% bovine serum albumin with $10 \%$ donkey serum (Sigma-Aldrich) in $1 \times$ TBS with $0.1 \%$ Tween 20. The brain slices were stained overnight in the blocking solution with the primary antibody [rabbit anti-cfos (1:500; Abcam, ab7963) and/or goat anti-CR (1:1000; Swant CG1)]. In the following day, the brain slices were first washed and then stained for $120 \mathrm{~min}$ with the secondary antibody [goat anti-rabbit conjugated to Alexa Flour 555 (1:500; Invitrogen, A21429), donkey anti-rabbit conjugated to Alexa Flour 488 (1:500; Invitrogen, A21206), or donkey anti-goat conjugated to Alexa Flour 647 (1:500; Abcam, ab150131)]. The brain slices were again washed and then mounted on glass slides
(Superfrost Plus, Thermo Scientific), immersed by 4',6-diamidino2-phenylindole containing ProLong Gold Antifade mounting solution (Invitrogen), and covered with borosilicate cover glass (VWR).

\section{Fluorescent in situ hybridization}

Mice were anesthetized with gas isoflurane. The brains were extracted and quickly frozen on aluminum foil over dry ice. Brains were kept overnight at $-80^{\circ} \mathrm{C}$. On the next day, the brains were equilibrated at $-20^{\circ} \mathrm{C}$ in the cryostat for at least $30 \mathrm{~min}$. Fresh frozen brain sections $(20 \mu \mathrm{m})$ containing the ZI were prepared and directly mounted onto glass slides. Selected slices were fixed for $30 \mathrm{~min}$ using 4\% PFA in PBS, dehydrated with increasing concentrations of ethanol $(50,70,100$, and $100 \%)$, and surrounded by hydrophobic barriers (ImmEdge, Vector Laboratories), before proceeding with the RNAscope Fluorescent Multiplex Assay (ACD, Bio-Techne) to label the mRNAs for Vgat (Slc32a1, ref319191), Vglut2 (Slc17a6, ref319171), SOM (SST, ref482691), CR (CALB2, ref313641), PV (Pvalb, ref421931), and CaMKII $\alpha$ (ref445231). Processed slides were immersed in ProLong Diamond Antifade mounting solution (Invitrogen) and covered with borosilicate cover glass.

\section{Imaging}

To visualize fluorescent immunohistochemistry, FISH, or reporter protein labeling in brain sections, mounted sections were imaged with a Zeiss LSM700 upright confocal microscope controlled by the ZEN Black image acquisition software (v2010; Zeiss). PLAN APO $\times 20$ (0.8 NA, air) and $\times 40(1.3 \mathrm{NA}$, oil) objectives were used to take lowand high-magnification images, respectively. Fixed wavelength $(405,488,555$, and $639 \mathrm{~nm})$ lasers were used to visualize specific fluorescent signals, while bright-field imaging was used for the anatomical features. For large throughput fluorescence imaging (anatomical tracing), mounted sections were imaged with a Zeiss Axio Scan.Z1 with a Fluar $\times 5(0.25 \mathrm{NA}$, air $)$ objective and filter sets suitable for imaging EGFP and tdTomato signals (38 and 43HE). Images were stitched with the acquisition software and further postprocessed in Fiji (v2.0.0, ImageJ).

\section{Behavior}

\section{General}

Mice were brought into the testing room from the housing facilities on the experimental day and acclimatized for at least an hour before starting any experimentation or experimental preparations, such as connecting optic fibers to fiber-implanted mice.

\section{Elevated rod exposure for cfos screening}

Control and experimental mice were group-housed in their home cages and were brought from the housing room into the testing room. A 60-min acclimation period was allowed before mice were tested or directly transcardially perfused. The remaining mice were placed on an elevated rod (100 cm tall and $3 \mathrm{~cm}$ in diameter) for $30 \mathrm{~min}$ and put back into the home cage for another $30 \mathrm{~min}$ before being perfused. It has been previously reported that increased cfos protein expression has been observed for as little as $30 \mathrm{~min}$ following stimulus exposure $(58,59)$. We chose a time frame that could detect changes in cfos expression and as close to the stimulus exposure as possible. Soft packing foam was placed around the bottom of the elevated rod. Mice that fell were placed back into the home cage and excluded.

\section{Elevated platform exposure for in vivo calcium imaging}

Mice were connected to the nVoke2 (Inscopix) system and recorded with the Inscopix data acquisition software with a $20-\mathrm{Hz}$ sampling rate while behaviorally recorded simultaneously from above using a 
camera (The Imaging Source) that is controlled by ANY-maze (v5.23, Stoelting) and sampled up to $20 \mathrm{~Hz}$. The mice were then placed in the enclosed center of the elevated platform and recorded while freely moving for $5 \mathrm{~min}$. The elevated platform is constructed by completely blocking the entrance of the closed arms of an EPM (arms with $7.5 \mathrm{~cm}$ in width, $30 \mathrm{~cm}$ in length separated by a center that is $7.5 \mathrm{~cm}$ in width and length and enclosed by $60-\mathrm{cm}$-tall cardboard pieces on the two sides not connected to the arms). This elevated platform maximizes and forces the recorded mice to explore the anxiogenic exposed open edges. The larger dimensions of this platform also provided more room for the recorded mice to maneuver.

\section{Optogenetic stimulation}

The light power for optogenetic stimulation (473 or $532 \mathrm{~nm}$ ) was adjusted with a power meter (Thorlabs) such that the illumination at the tip of the optic fibers would be at around $10 \mathrm{~mW}$. To sufficiently activate ZI neurons with ChR2, 5-ms pulses were provided at $40 \mathrm{~Hz}(473 \mathrm{~nm})$. Constant light $(532 \mathrm{~nm})$ stimulation was used to drive the Arch3.0-mediated inhibition. The number of light stimulation periods was counterbalanced with periods where no light was provided in an alternating manner over 6 min with 1-min bins.

\section{Elevated O-maze}

Cannula-implanted mice that received intracranial infusion of either diazepam or vehicle (see the "Surgery" or "Diazepam infusion" section), or fiber-implanted mice that were attached to the laser cables (see the "Behavior" or "Optogenetic stimulation" section) were placed on the open arm of the EOM (arms with $5 \mathrm{~cm}$ in width; the maze is $55 \mathrm{~cm}$ in diameter and $60 \mathrm{~cm}$ above the ground) facing toward the closed arm (enclosed by two 15-cm-tall gray opaque plastic walls). The translucent floor allowed a camera mounted below facing upward to record the shadow of the tested mice as it traveled along the EOM. Videos were recorded and tracked with ANY-maze at a rate of up to $30 \mathrm{~Hz}$ for either $10 \mathrm{~min}$ (infusion animals) or $6 \mathrm{~min}$ (optogenetics animals).

\section{Open field test}

Mice were placed in the center of a rectangular OFT box $(30 \mathrm{~cm}$ in width and $45 \mathrm{~cm}$ in length, with 30 -cm-tall walls) for either $10 \mathrm{~min}$ (infusion animals) or 6 min (optogenetics animals). Videos were recorded with a downward-facing camera from above with ANY-maze in the same manner as mentioned previously.

\section{Unique behaviors in the closed chamber}

Mice were placed in the center of a transparent plexiglass rectangular box (25 cm in width and $20 \mathrm{~cm}$ in length, with $15-\mathrm{cm}$-tall walls) with a closed ceiling that had a circular $(5 \mathrm{~cm}$ in diameter) hole that let out the fiber cables but not the mice for $6 \mathrm{~min}$. Videos were recorded with a front-facing camera from the side with ANY-maze in the same manner as mentioned previously. To test the extent of activated neurons following laser stimulation, a cohort of bilaterally implanted Vglut2-ChR2-expressing mice were stimulated in the closed chamber as mentioned above, returned to the home cage, transcardially perfused after $60 \mathrm{~min}$, and followed up with a post hoc cfos immunohistochemistry staining.

\section{Analysis \\ Calcium imaging}

The position of the center of the video recorded the mouse, and its movement speed was extracted from ANY-maze together with the digital boundaries of the center and the open arms of the elevated platform. The behavioral trace was then exported to MATLAB (v2018b, MathWorks) for further analysis.
Calcium transients recorded from the nVoke2 system were preprocessed in the Inscopix Data Processing Software (v1.3, Inscopix). The time-stamped calcium traces were also exported to MATLAB. Calcium transients and the behavioral traces were first aligned to each other. Calcium traces were denoised and standardized. Calcium and behavior traces were then both binned to 500-ms windows. To examine the relationship between anxiety and the neuronal activity in the ZI, correlational analyses were made between the distance of the recorded mice to the enclosed center (center distance) and the calcium transients of the recorded cells. Center distance is an assessment for anxiety because the further the animal is from the center, the more exposed the animal is and, thus, the higher the level of anxiety. The center distance is calculated by subtracting the lengthwise position of the center of the animal from that of the center of the platform and taking the absolute value. Pearson's correlation coefficient was calculated from the distribution of the binned calcium activity and center distance values. To characterize cells as being positively, negatively, or not correlated between these two parameters, a bootstrap test was performed by randomly shifting the calcium traces 1000 times in a circular manner and then calculating the correlation coefficient. Cells whose real correlation coefficient was negative and below the 5 th percentile or positive and above the 95 th percentile of the random correlation coefficients were classified as negatively or positively correlated, respectively. Cells that did not fall into this range were classified as not correlated.

To further show that these correlated cells displayed activity with respect to the level of anxiety that the recorded mice experienced, the difference in activity between the time when the animals was in the center and the edges (defined as an area on extremities of the open arm with the same size as the center) was calculated for each cell and compared across groups. All recorded animals spent more time in the center as compared to the edges. To reduce sampling bias, the activity in the center was randomly sampled 1000 times to match the number of bins the animals spent in the edges, and the averaged difference was reported.

To examine whether the correlated cells displayed changes in activity as the recorded animal transitioned between areas that are less anxiogenic to more anxiogenic and vice versa, calcium transients surrounding ( $2.5 \mathrm{~s}$ before and after) the entry or the exit of the edge or the center were extracted, grouped, and compared. To visualize changes across cells and groups, transients were adjusted such that the average activity in the baseline window before the change in compartment is 0 . The delta responses following each compartment change between positively, negatively, and not correlated cells were extracted and compared.

To show that the center distance-correlated cells encoded anxiety rather than movement speed, the same correlation analysis was applied between calcium transients and movement speed for each cell. For each categorized (positively, negatively, and not correlated) center distance-correlated cell groups, the proportion of each of the three categories of movement speed-correlated cells were shown.

\section{Behavioral paradigms}

All parameters were extracted from ANY-maze (EOM and OFT). Unique behaviors such as jumps and rears were visually identified and manually quantified.

\section{Statistics}

All statistical comparisons, unless otherwise noted, were analyzed with Prism (v8.4.3; GraphPad). The significance level was set at 5\% $(\alpha=0.05)$ in all cases. Student's $t$ test was used to make comparisons 
between two groups of data. The unpaired design was used when the samples were independent from one another (number of cfos-labeled ZI cells prepared from mice that were exposed to the elevated anxious experience or the control home conditions), while the paired design was applied in cases where repeated measures were made (activity of center distance cells before and after compartment change, effect of diazepam or vehicle on the performance in the EOM and OFT of the same mice, effect of diazepam on the recorded inhibitory currents onto ZI cells with respect to the baseline period before, and effect of photoactivation of CR- and Vglut2-ZI cells to respectively trigger rears and jumps as compared to periods in the same session where the lights were off). One-way analysis of variance (ANOVA) with Bonferroni's multiple comparison tests were used to compare the differences across multiple (greater than two) independent samples (differences in activity between the center and the edge across the different center distance-correlated groups; electrophysiological properties among the SOM-, CR-, and Vglut2-expressing ZI cells; and the differences in behavioral parameters among control, ChR2expressing, and Arch-expressing groups).

\section{SUPPLEMENTARY MATERIALS}

Supplementary material for this article is available at https://science.org/doi/10.1126/ sciadv.abf6709

View/request a protocol for this paper from Bio-protocol.

\section{REFERENCES AND NOTES}

1. D. C. Blanchard, R. J. Blanchard, Defensive behaviors, fear, and anxiety, in Handbook of Anxiety and Fear, vol. 17 of Handbook of Behavioral Neuroscience, R. J. Blanchard, D. C. Blanchard, G. Griebel, D. Nutt, Eds. (Handbook of Behavioral Neuroscience Series, Elsevier, 2008), chap. 2.4, pp.63-79; www.sciencedirect.com/science/article/pii/ S1569733907000057.

2. P. Tovote, J. P. Fadok, A. Lüthi, Neuronal circuits for fear and anxiety. Nat. Rev. Neurosci. 16, 317-331 (2015).

3. K. M. Tye, R. Prakash, S.-Y. Kim, L. E. Fenno, L. Grosenick, H. Zarabi, K. R. Thompson, V. Gradinaru, C. Ramakrishnan, K. Deisseroth, Amygdala circuitry mediating reversible and bidirectional control of anxiety. Nature 471, 358-362 (2011).

4. A. C. Felix-Ortiz, A. Beyeler, C. Seo, C. A. Leppla, C. P. Wildes, K. M. Tye, BLA to vHPC inputs modulate anxiety-related behaviors. Neuron 79, 658-664 (2013).

5. S.-Y. Kim, A. Adhikari, S. Y. Lee, J. H. Marshel, C. K. Kim, C. S. Mallory, M. Lo, S. Pak, J. Mattis, B. K. Lim, R. C. Malenka, M. R. Warden, R. Neve, K. M. Tye, K. Deisseroth, Diverging neural pathways assemble a behavioural state from separable features in anxiety. Nature $\mathbf{4 9 6}$ 219-223 (2013).

6. J. H. Jennings, D. R. Sparta, A. M. Stamatakis, R. L. Ung, K. E. Pleil, T. L. Kash, G. D. Stuber, Distinct extended amygdala circuits for divergent motivational states. Nature 496, 224-228 (2013).

7. A. Adhikari, M. A. Topiwala, J. A. Gordon, Single units in the medial prefrontal cortex with anxiety-related firing patterns are preferentially influenced by ventral hippocampal activity. Neuron 71, 898-910 (2011).

8. N. Padilla-Coreano, S. S. Bolkan, G. M. Pierce, D. R. Blackman, W. D. Hardin, A. L. Garcia-Garcia, T. J. Spellman, J. A. Gordon, Direct ventral hippocampal-prefrontal input is required for anxiety-related neural activity and behavior. Neuron 89, 857-866 (2016).

9. J. C. Jimenez, K. Su, A. R. Goldberg, V. M. Luna, J. S. Biane, G. Ordek, P. Zhou, S. K. Ong, M. A. Wright, L. Zweifel, L. Paninski, R. Hen, M. A. Kheirbek, Anxiety cells in a hippocampalhypothalamic circuit. Neuron 97, 670-683.e6 (2018).

10. R. M. Cassidy, Y. Lu, M. Jere, J.-B. Tian, Y. Xu, L. R. Mangieri, B. Felix-Okoroji, J. Selever, Y. Xu, B. R. Arenkiel, Q. Tong, A lateral hypothalamus to basal forebrain neurocircuit promotes feeding by suppressing responses to anxiogenic environmental cues. Sci. Adv. 5, eaav1640 (2019).

11. A. M. Burrows, P. D. Ravin, P. Novak, M. L. B. Peters, B. Dessureau, J. Swearer, J. G. Pilitsis, Limbic and motor function comparison of deep brain stimulation of the zona incerta and subthalamic nucleus. Neurosurgery 70, ons125-ons130 (2012).

12. A. Gourisankar, S. A. Eisenstein, N. T. Trapp, J. M. Koller, M. C. Campbell, M. Ushe, J. S. Perlmutter, T. Hershey, K. J. Black, Mapping movement, mood, motivation and mentation in the subthalamic nucleus. R. Soc. Open Sci. 5, 171177 (2018).

13. J. Chen, A. R. Kriegstein, A GABAergic projection from the zona incerta to cortex promotes cortical neuron development. Science 350, 554-558 (2015).
14. M. K. Sanghera, J. Anselmo-Franci, S. M. McCann, Effect of medial zona incerta lesions on the ovulatory surge of gonadotrophins and prolactin in the rat. Neuroendocrinology 54, 433-438 (1991).

15. J. F. Murray, J. G. Mercer, R. A. H. Adan, J. Datta, C. Aldairy, K. M. Moar, B. I. Baker, M. J. Stock, C. A. Wilson, The effect of leptin on luteinizing hormone release is exerted in the zona incerta and mediated by melanin-concentrating hormone. J. Neuroendocrinol. 12, 1133-1139 (2000)

16. L. L. Walsh, S. P. Grossman, Zona incerta lesions impair osmotic but not hypovolemic thirst. Physiol. Behav. 16, 211-215 (1976).

17. B. A. Stamoutsos, R. G. Carpenter, L. Grossman, S. P. Grossman, Impaired feeding responses to intragastric, intraperitoneal, and subcutaneous injections of 2-deoxy-Dglucose in rats with zona incerta lesions. Physiol. Behav. 23, 771-776 (1979).

18. X. Zhang, A. N. van den Pol, Rapid binge-like eating and body weight gain driven by zona incerta GABA neuron activation. Science 356, 853-859 (2017).

19. M. Liu, C. Blanco-Centurion, R. Konadhode, S. Begum, D. Pelluru, D. Gerashchenko, T. Sakurai, M. Yanagisawa, A. N. van den Pol, P. J. Shiromani, Orexin gene transfer into zona incerta neurons suppresses muscle paralysis in narcoleptic mice. J. Neurosci. 31, 6028-6040 (2011).

20. K. Liu, J. Kim, D. W. Kim, Y. S. Zhang, H. Bao, M. Denaxa, S.-A. Lim, E. Kim, C. Liu, I. R. Wickersham, V. Pachnis, S. Hattar, J. Song, S. P. Brown, S. Blackshaw, Lhx6-positive GABA-releasing neurons of the zona incerta promote sleep. Nature 548, 582-587 (2017).

21. J. C. Trageser, A. Keller, Reducing the uncertainty: Gating of peripheral inputs by zona incerta. J. Neurosci. 24, 8911-8915 (2004).

22. N. Urbain, M. Deschênes, Motor cortex gates vibrissal responses in a thalamocortical projection pathway. Neuron 56, 714-725 (2007).

23. R. Masri, R. L. Quiton, J. M. Lucas, P. D. Murray, S. M. Thompson, A. Keller, Zona incerta: A role in central pain. J. Neurophysiol. 102, 181-191 (2009).

24. W. W. Kaelber, T. B. Smith, Projections of the zona incerta in the cat, with stimulation controls. Exp. Neurol. 63, 177-200 (1979).

25. F. Benedetti, L. Colloca, M. Lanotte, B. Bergamasco, E. Torre, L. Lopiano, Autonomic and emotional responses to open and hidden stimulations of the human subthalamic region. Brain Res. Bull. 63, 203-211 (2004).

26. L. V. Loskutova, I. M. Vinnitskii, I. RYu, Participation of the zona incerta in mechanisms of the conditioned avoidance reaction. Neurosci. Behav. Physiol. 11, 60-62 (1981).

27. M. Zhou, Z. Liu, M. D. Melin, Y. H. Ng, W. Xu, T. C. Südhof, A central amygdala to zona incerta projection is required for acquisition and remote recall of conditioned fear memory. Nat. Neurosci. 21, 1515-1519 (2018).

28. X.-I. Chou, X. Wang, Z.-g. Zhang, L. Shen, B. Zingg, J. Huang, W. Zhong, L. Mesik, L. I. Zhang, H. W. Tao, Inhibitory gain modulation of defense behaviors by zona incerta. Nat. Commun. 9, 1151 (2018).

29. C. Kolmac, J. Mitrofanis, Distribution of various neurochemicals within the zona incerta: An immunocytochemical and histochemical study. Anat. Embryol. 199, 265-280 (1999).

30. J. Mitrofanis, K. Ashkan, B. A. Wallace, A.-L. Benabid, Chemoarchitectonic heterogeneities in the primate zona incerta: Clinical and functional implications. J. Neurocytol. 33, 429-440 (2004).

31. C. Watson, C. R. P. Lind, M. G. Thomas, The anatomy of the caudal zona incerta in rodents and primates. J. Anat. 224, 95-107 (2014)

32. G. Griebel, A. Holmes, 50 years of hurdles and hope in anxiolytic drug discovery. Nat. Rev. Drug Discov. 12, 667-687 (2013).

33. R. J. Rodgers, A. Dalvi, Anxiety, defence and the elevated plus-maze. Neurosci. Biobehav. Rev. 21, 801-810 (1997).

34. A. A. Walf, C. A. Frye, The use of the elevated plus maze as an assay of anxiety-related behavior in rodents. Nat. Protoc. 2, 322-328 (2007).

35. J. K. Shepherd, S. S. Grewal, A. Fletcher, D. J. Bill, C. T. Dourish, Behavioural and pharmacological characterisation of the elevated "zero-maze" as an animal model of anxiety. Psychopharmacology 116, 56-64 (1994).

36. C. Belzung, G. Griebel, Measuring normal and pathological anxiety-like behaviour in mice: A review. Behav. Brain Res. 125, 141-149 (2001).

37. S. B. Sartori, R. Landgraf, N. Singewald, The clinical implications of mouse models of enhanced anxiety. Future Neurol. 6, 531-571 (2011).

38. L. M. Behlke, R. A. Foster, J. Liu, D. Benke, R. S. Benham, A. J. Nathanson, B. K. Yee, H. U. Zeilhofer, E. Engin, U. Rudolph, A pharmacogenetic "restriction-of-function" approach reveals evidence for anxiolytic-like actions mediated by $\alpha 5$-containing $\mathrm{GABA}_{\mathrm{A}}$ receptors in mice. Neuropsychopharmacology 41, 2492-2501 (2016).

39. J. N. Crawley, Neuropharmacologic specificity of a simple animal model for the behavioral actions of benzodiazepines. Pharmacol. Biochem. Behav. 15, 695-699 (1981).

40. S. A. Krömer, M. S. Keßler, D. Milfay, I. N. Birg, M. Bunck, L. Czibere, M. Panhuysen, B. Pütz, J. M. Deussing, F. Holsboer, R. Landgraf, C. W. Turck, Identification of glyoxalase-I as a protein marker in a mouse model of extremes in trait anxiety. J. Neurosci. $\mathbf{2 5}$ 4375-4384 (2005). 
41. H. Hu, Y. Cui, Y. Yang, Circuits and functions of the lateral habenula in health and in disease. Nat. Rev. Neurosci. 21, 277-295 (2020).

42. I. Lazaridis, O. Tzortzi, M. Weglage, A. Märtin, Y. Xuan, M. Parent, Y. Johansson, J. Fuzik, D. Fürth, L. E. Fenno, C. Ramakrishnan, G. Silberberg, K. Deisseroth, M. Carlén, K. Meletis, A hypothalamus-habenula circuit controls aversion. Mol. Psychiatry 24, 1351-1368 (2019).

43. A. Venkataraman, N. Brody, P. Reddi, J. Guo, D. Gordon Rainnie, B. G. Dias, Modulation of fear generalization by the zona incerta. Proc. Natl. Acad. Sci. U.S.A. 116, 9072-9077 (2019).

44. H. Won, H.-R. Lee, H. Y. Gee, W. Mah, J.-I. Kim, J. Lee, S. Ha, C. Chung, E. S. Jung, Y. S. Cho, S.-G. Park, J.-S. Lee, K. Lee, D. Kim, Y. C. Bae, B.-K. Kaang, M. G. Lee, E. Kim, Autistic-like social behaviour in Shank2-mutant mice improved by restoring NMDA receptor function. Nature 486, 261-265 (2012).

45. K. R. Stover, S. Lim, T.-L. Zhou, P. M. Stafford, J. Chow, H. Li, N. Sivanenthiran, S. Mylvaganam, C. Wu, D. F. Weaver, J. Eubanks, L. Zhang, Susceptibility to hippocampal kindling seizures is increased in aging C57 black mice. IBRO Rep. 3, 33-44 (2017).

46. J. P. Fadok, S. Krabbe, M. Markovic, J. Courtin, C. Xu, L. Massi, P. Botta, K. Bylund, C. Müller, A. Kovacevic, P. Tovote, A. Lüthi, A competitive inhibitory circuit for selection of active and passive fear responses. Nature 542, 96-100 (2017).

47. P. Tovote, M. S. Esposito, P. Botta, F. Chaudun, J. P. Fadok, M. Markovic, S. B. E. Wolff, C. Ramakrishnan, L. Fenno, K. Deisseroth, C. Herry, S. Arber, A. Lüthi, Midbrain circuits for defensive behaviour. Nature 534, 206-212 (2016).

48. C. Lever, S. Burton, J. O'Keefe, Rearing on hind legs, environmental novelty, and the hippocampal formation. Rev. Neurosci. 17, 111-133 (2006).

49. O. Sturman, P.-L. Germain, J. Bohacek, Exploratory rearing: A context- and stress-sensitive behavior recorded in the open-field test. Stress 21, 443-452 (2018).

50. Z.-d. Zhao, Z. Chen, X. Xiang, M. Hu, H. Xie, X. Jia, F. Cai, Y. Cui, Z. Chen, L. Qian, J. Liu, C. Shang, Y. Yang, X. Ni, W. Sun, J. Hu, P. Cao, H. Li, W. L. Shen, Zona incerta GABAergic neurons integrate prey-related sensory signals and induce an appetitive drive to promote hunting. Nat. Neurosci. 22, 921-932 (2019).

51. H. Taniguchi, M. He, P. Wu, S. Kim, R. Paik, K. Sugino, D. Kvitsani, Y. Fu, J. Lu, Y. Lin G. Miyoshi, Y. Shima, G. Fishell, S. B. Nelson, Z. J. Huang, A resource of $\mathrm{Cre}$ driver lines for genetic targeting of GABAergic neurons in cerebral cortex. Neuron 71, 995-1013 (2011).

52. L. Vong, C. Ye, Z. Yang, B. Choi, S. Chua Jr., B. B. Lowell, Leptin action on GABAergic neurons prevents obesity and reduces inhibitory tone to POMC neurons. Neuron 71, 142-154 (2011).

53. L. Madisen, T. A. Zwingman, S. M. Sunkin, S. W. Oh, H. A. Zariwala, H. Gu, L. L. Ng, R. D. Palmiter, M. J. Hawrylycz, A. R. Jones, E. S. Lein, H. Zeng, A robust and high-throughput
Cre reporting and characterization system for the whole mouse brain. Nat. Neurosci. 13 133-140 (2010).

54. F. B. J. Keith, G. Paxinos, The Mouse Brain in Stereotaxic Coordinates (Elsevier Inc., ed. 3 , 2007).

55. M. N. Cook, M. Crounse, L. Flaherty, Anxiety in the elevated zero-maze is augmented in mice after repeated daily exposure. Behav. Genet. 32, 113-118 (2002).

56. A. P. Carobrez, L. J. Bertoglio, Ethological and temporal analyses of anxiety-like behavior: The elevated plus-maze model 20 years on. Neurosci. Biobehav. Rev. 29, 1193-1205 (2005).

57. D. R. Sparta, A. M. Stamatakis, J. L. Phillips, N. Hovelsø, R. van Zessen, G. D. Stuber, Construction of implantable optical fibers for long-term optogenetic manipulation of neural circuits. Nat. Protoc. 7, 12-23 (2011).

58. A. Chaudhuri, S. Zangenehpour, F. Rahbar-Dehgan, F. Ye, Molecular maps of neural activity and quiescence. Acta Neurobiol. Exp. (Wars) 60, 403-410 (2000).

59. X. Lin, C. A. Itoga, S. Taha, M. H. Li, R. Chen, K. Sami, F. Berton, W. Francesconi, X. Xu, c-Fos mapping of brain regions activated by multi-modal and electric foot shock stress. Neurobiol. Stress 8, 92-102 (2018).

Acknowledgments: We thank the past and current members of the Tan laboratory for constructive input, the Biozentrum Imaging Facility for microscopy training, and the Inscopix team for technical support of the calcium imaging experiment. Funding: This work was supported by the Swiss National Science Foundation grants PPOOP3_150683 and BSSGIO_155830. Author contributions: Z.L., G.R., and K.R.T. all contributed to the experimental design. G.R. conducted the calcium imaging experiments. K.R.T. conducted the electrophysiology experiments. Z.L. conducted all other experiments. Z.L. and K.R.T. analyzed the data and prepared the manuscript. Competing interests: The authors declare that they have no competing interests. Data and materials availability: All data needed to evaluate the conclusions in the paper are present in the paper and/or the Supplementary Materials.

Submitted 11 November 2020

Accepted 20 July 2021

Published 10 September 2021

$10.1126 /$ sciadv abf6709

Citation: Z. Li, G. Rizzi, K. R. Tan, Zona incerta subpopulations differentially encode and modulate anxiety. Sci. Adv. 7, eabf6709 (2021) 